\title{
Wild-type sTREM2 blocks $A \beta$ aggregation and neurotoxicity, while the Alzheimer's R47H mutant does the opposite
}

Anna Vilalta ${ }^{1 \dagger}$, Ye Zhou ${ }^{2 \dagger}$, Jean Sevalle ${ }^{2 \dagger}$, Jennifer K. Griffin ${ }^{2 \dagger}$, Kanayo Satoh ${ }^{2 \dagger}$, David H.

Allendorf $^{1}$, Suman De ${ }^{4}$, Mar Puigdellívol ${ }^{1}$, Arturas Bruzas ${ }^{1}$, Miguel A. Burguillos ${ }^{1,3}$, Roger B.

Dodd $^{3}$, Fusheng Chen ${ }^{2}$, Yalun Zhang ${ }^{2}$, Patrick Flagmeier ${ }^{4}$, Lisa-Maria Needham ${ }^{4}$, Masahiro

Enomoto $^{5}$, Seema Qamar ${ }^{3}$, James Henderson ${ }^{3}$, Jochen Walter ${ }^{6}$, Paul E. Fraser ${ }^{2}$, David

Klenerman ${ }^{4}$, Steven F. Lee ${ }^{4}$, Peter St George-Hyslop ${ }^{2,3^{*}}$, Guy C. Brown ${ }^{1^{*}}$

1. Department of Biochemistry, University of Cambridge, Tennis Court Road, Cambridge CB2 $1 \mathrm{QW}$, U.K.

2. Departments of Medicine (Neurology) and Medical Biophysics, University of Toronto and University Health Network, Krembil Discovery Tower, 60 Leonard Avenue, Toronto, ON, M5T 0S8, Canada.

3. Cambridge Institute for Medical Research, Keith Peters Building, Room 4.36, Cambridge Biomedical Campus, Hills Road, Cambridge CB2 OXY, U.K.

4. Department of Chemistry, University of Cambridge, Lensfield Rd, Cambridge CB2 1EW, U.K.

5. Princess Margaret Cancer Centre, University Health Network, Department of Medical Biophysics, University of Toronto, Toronto, ON, Canada.

6. Molecular Cell Biology, Department of Neurology, University of Bonn, Venusberg Campus 1, 53105 Bonn, Germany.

Length: 25,000 characters, including spaces, excluding references and methods.

† Contributed equally.

* To whom correspondence should be addressed: gcb3@cam.ac.uk or phs22@cam.ac.uk 


\begin{abstract}
Missense mutations (e.g. R47H) of the microglial receptor TREM2 increase risk of Alzheimer's disease (AD), and the soluble ectodomain of wild-type TREM2 (sTREM2) appears to protect in vivo, but the underlying mechanisms are unclear. We show that $A \beta$ oligomers bind to TREM2, inducing shedding of sTREM2. Wild-type sTREM2 inhibits $A \beta$ oligomerization, fibrillization and neurotoxicity, and disaggregates preformed $A \beta$ oligomers and protofibrils. In contrast, the $\mathrm{R} 47 \mathrm{H}$ $A D$-risk variant of STREM2 is less able to bind and disaggregate oligomeric $A \beta$, but rather promotes $A \beta$ protofibril formation and neurotoxicity. Thus, in addition to mediating phagocytosis, wild-type TREM2 may protect against amyloid pathology by A $\beta$-induced release of sTREM2 that blocks $A \beta$ aggregation and neurotoxicity; while $\mathrm{R} 47 \mathrm{H}$ sTREM2 promotes $A \beta$ aggregation into neurotoxic forms, which may explain why the $\mathrm{R} 47 \mathrm{H}$ variant gene increases $A D$ risk several fold.
\end{abstract}

Keywords: Amyloid beta, Alzheimer's disease, TREM2, microglia, oligomers, neurotoxicity, sTREM2.

\title{
Introduction
}

A prominent neuropathological feature of Alzheimer's disease (AD) is the presence of extracellular deposits of the amyloid $\beta$-peptide in amyloid plaques, surrounded by activated microglia ${ }^{1-3}$. The importance of this microglial response to the pathogenesis of AD has been highlighted by the recent discovery of sequence variants in multiple genes expressed in microglia that alter risk for AD. Prominent amongst these microglial AD-risk genes is the "triggering receptor expressed on myeloid cells 2" (TREM2) ${ }^{4-6}$, of which there are several missense mutations in the ectodomain, including $\mathrm{R} 47 \mathrm{H}$, associated with increased risk for $A D^{4-6}$. The biological mechanisms underlying this association remain unclear.

Full length TREM2 is expressed on the plasma membrane of microglia, where it can be cleaved by one or more metalloproteases to produce i) a membrane-bound C-terminal fragment (CTF); and ii) an N-terminal fragment consisting of the soluble ectodomain of TREM2 (sTREM2), which is released into the extracellular space ${ }^{7-9}$. sTREM2 has been thought of as a non-functional, degradation product of TREM2, and used as a biomarker of microglial activation $^{10-12}$. However, several recent observations suggest the possibility that STREM2 per se may play a role protecting against $A D$ by interacting with $A \beta$. First, oligomeric $A \beta$ binds to TREM2 and to sTREM2-Fc fusion protein ${ }^{13-15}$, suggesting that sTREM2 might bind $A \beta$ and 
potentially affect its aggregation state. Second, injection or expression of sTREM2 in the hippocampus of 5xFAD mice reduces both amyloid plaque load and memory deficits ${ }^{16}$, indicating that sTREM2 inhibits amyloid pathology somehow. Third, in transgenic mouse models overproducing $A \beta$, the knockout of TREM2 expression accelerates amyloid plaque seeding ${ }^{17}$ and the plaques have increased protofibrillar halos and hotspots ${ }^{3,16,18}$, indicating that either TREM2 or sTREM2 inhibit plaque formation. Fourth, in the earliest pre-symptomatic stages of $A D$, at the time of $A \beta$ deposition, sTREM2 levels in cerebrospinal fluid (CSF) are lower than in healthy controls ${ }^{10,19}$, consistent with sTREM2 being an endogenous inhibitor of $A \beta$ deposition. However, the CSF $A \beta$ levels rise in the early symptomatic stages of $A D$, and then decline again at later stages of $A D^{11,12}$. Fifth, mild cognitive impairment $(\mathrm{MCl})$ and $A D$ patients with higher sTREM2 levels in CSF have slower brain atrophy, cognitive decline and clinical decline ${ }^{20,21}$, consistent with sTREM2 inhibiting AD progression. Sixth, healthy controls and $\mathrm{MCl}$ patients with higher STREM2 levels in CSF have slower progression of amyloid and tau deposition ${ }^{22}$, consistent with sTREM2 inhibiting $A \beta$ aggregation and subsequent tau pathology.

All of these in vivo findings are compatible with the hypothesis that sTREM2 might protect against $A D$, potentially by impacting $A \beta$ aggregation. To explore this hypothesis and the mechanisms involved, we investigated the interaction of $A \beta$ with sTREM2 in vitro. We report that soluble $A \beta$ oligomers bind TREM2 receptor on microglia and induce shedding of sTREM2. Next, we show that sTREM 2 can bind and disaggregate $A \beta$ oligomers, block $A \beta$ fibrillization and reduce $A \beta$ neurotoxicity. These activities are attenuated in the $R 47 H$ TREM2 holoprotein and R47H sTREM2. Moreover, the R47H sTREM2 promotes formation of morphologically distinct $A \beta$ protofibrils. These data indicate additional mechanisms by which TREM2 protects against Alzheimer's disease, and a previously unrecognised mechanism by which the $\mathrm{R} 47 \mathrm{H}$ mutant increases risk.

\section{Results}

\section{$A \beta$ oligomers bind TREM2 holoprotein and induce TREM2 ectodomain shedding}

To confirm that TREM2 can act as a cell surface receptor for $A \beta$ oligomers, we treated mouse primary microglia or TREM2-transfected HeLa cells with A 342 oligomers that have been characterized both by electron microscopy and by oligomer/fibril specific antibodies (Supplementary Fig. 1; note only $A \beta 42$ was used in this work and will be referred to as $A \beta$ henceforth). We then immunoprecipitated TREM2 from lysates of these cells, and found that $A \beta$ 
co-immunoprecipitated with endogenous TREM2 on primary microglia from wild-type mice, but not on microglia from TREM2-- knockout mice (Supplementary Fig. 2i). The A $\beta$ binding was prevented by TREM2-blocking antibody (Supplementary Fig. 2ii). Oligomeric A $\beta$ bound to TREM2 but not TREM1 (Supplementary Fig. 2iii). Monomeric A $\beta$ co-immunoprecipitated with TREM2 less efficiently than oligomeric $A \beta$ (Supplementary Fig. 2iv and $v, p=0.03$ ). The TREM2: $A \beta$ oligomer interaction is at least partially specific because neither of two other neurodegeneration-associated oligomeric proteins (oligomeric $\alpha$-synuclein or oligomeric Tau) bound to TREM2 even in HeLa cells overexpressing TREM2 and DAP12 (Supplementary Fig. 3 ). These results, confirm and extend the work of other groups showing that oligomeric $A \beta$ binds TREM2 ${ }^{13-15}$.

We next tested the consequences of $A \beta$ oligomers binding to TREM2 on primary microglia. $A \beta$ oligomers induced release of sTREM2 into the medium of primary microglia from wild-type mice, but not microglia from mice engineered to express R47H TREM2 (Supplementary Fig. 4i, p 0.05). To study this effect in more detail, we stably expressed TREM2 (together with DAP12) in HEK293 cells. Treatment of these cells with A $\beta$ oligomers resulted in dose-dependent release of STREM2 into the medium and the accumulation of TREM2-CTF in cell lysates (Figures $1 \mathrm{i} \&$ ii \& Supplementary Fig. 4ii). In contrast, treatment with $A \beta$ monomers or fibrils resulted in no modulation of sTREM2's release (Fig. 1iii \& Supplementary Fig. 5). The endoproteolysis of TREM2 induced by oligomeric $A \beta$ was attenuated in HEK293 cells expressing R47H TREM2 (Fig. 1ii \& Supplementary Fig. 4ii \& Fig. 5). Thus, oligomeric $A \beta$ stimulates shedding of sTREM2 from wild-type TREM2, but less so from R47H TREM2. 


\section{Figure 1}

i)

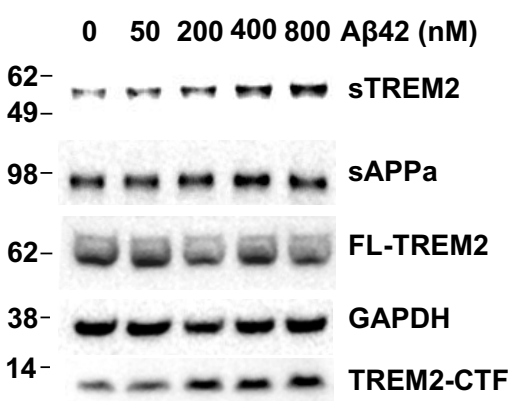

iv)

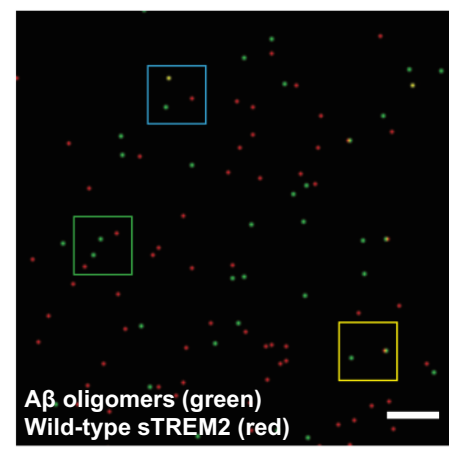

ii)

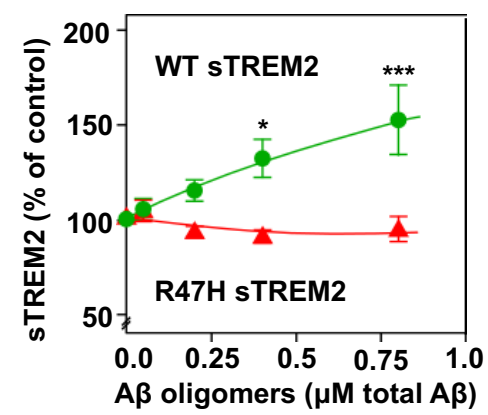

v)

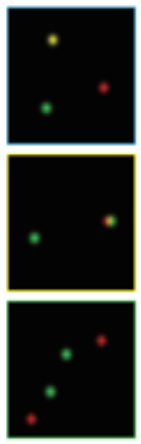

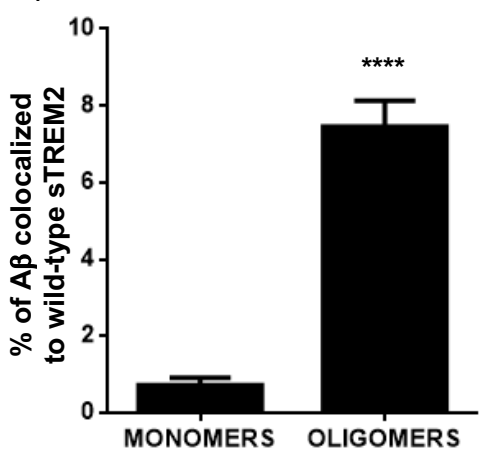

iii)

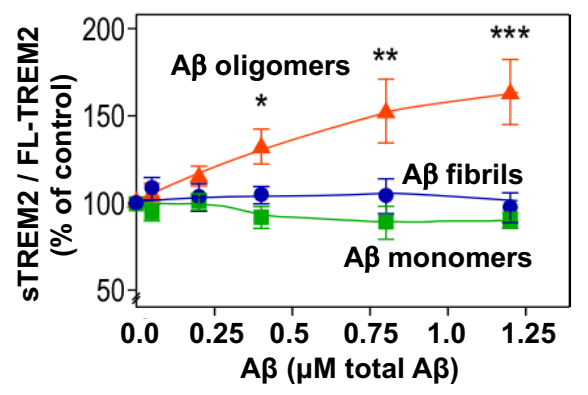

vi)

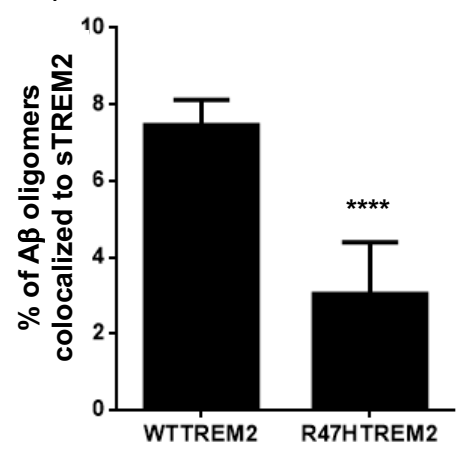

FIGURE 1. A oligomers induce TREM2 proteolysis and sTREM2 release, which then binds $A \beta$ oligomers, but R47H sTREM2 binds less.

i) Western blot of cell lysate and supernatant (sTREM2) of HEK293 cells co-expressing DAP12 and full-length TREM2 (FL-TREM2)16 hours after adding A $\beta$ oligomers. ii) Quantification of sTREM2 release from wild-type (green line) and R47H TREM2 (red line) expressing HEK293 cells. iii) Quantification of sTREM2 release from wild-type TREM2 expressing HEK293 cells induced by doses of $A \beta$ oligomers (red line), monomers (green line) or fibrils (blue line). For both ii \& iii) error bars $=$ SEM; ${ }^{*}=p<0.05^{* *}=p<0.01^{* * *}=p<0.001, n=3$ independent experiments; one-way ANOVA with Tukey's post-hoc multiple comparisons test. iv) Example field of singlemolecule TIRF imaging of mixture of $A \beta$ oligomers (green) and wild-type TREM2 ectodomain (red), where co-localised spots appear yellow. Scale bar: 1 micron. Magnified image of three sections of field at right. v) Proportion of monomeric or oligomeric $A \beta$ colocalized with wildtype sTREM2. vi) Proportion of $A \beta$ oligomers colocalized with wild-type or R47H TREM2 ectodomain. For v) \& vi), error bars = SEM; ${ }^{* * * *} p<0.0001, n=3$ independent preparations, each analysed in 9 fields each; two tailed $t$ test of significance. 


\section{Wild-type sTREM2 binds A $\beta$ oligomers better than R47H TREM2}

Because $A \beta$ oligomers bind to TREM2 and induce shedding of STREM2, it is of interest whether sTREM2 itself binds $A \beta$ oligomers. Three groups reported that wild-type sTREM2 fused to the Fc domain of $\operatorname{lgG}$ binds oligomeric $A \beta^{13-15}$. However, Zhao et $a l^{13}$ and Zhong et al ${ }^{14}$ found that R47H sTREM2-Fc bound less than wild-type to $A \beta$, while Lessard et al ${ }^{15}$ found that they bound equally. We applied three orthogonal assays using an approach that did not require fusion of sTREM2 to the Fc domain of lgG, to reassess this question. Firstly, we examined this interaction at the molecular level using single molecule total internal reflection fluorescence (TIRF) microscopy-based two-colour coincidence detection ${ }^{23}$. HiLyte 488-dye labelled A $\beta$ was oligomerized and TAMRA-dye labelled STREM2 added and imaged. We took advantage of the fact that monomeric $A \beta$ bleaches rapidly, so co-localization of $A \beta$ and sTREM2 before and after bleaching enabled us to distinguish between monomers and oligomers. These experiments revealed that wild-type sTREM2 bound oligomeric $A \beta$ much more than monomeric $A \beta$ (Fig. 1iv $\& v$ ), and that R47H sTREM2 bound less well to oligomeric $A \beta$ than wild-type sTREM2 did (Fig. $1 \mathrm{vi)}$.

Second, this result was confirmed by a semi-quantitative dot blot assay, which showed that wild-type sTREM2 preferentially bound oligomeric $A \beta$ over monomeric $A \beta$, and that $R 47 \mathrm{H}$ sTREM2 bound less of both forms of $A \beta$ (Supplementary Fig. $6 i$ \& ii).

Third, we used Bio-Layer Interferometry $(B L I)^{24}$ to quantitatively assess the $A \beta$ oligomer: sTREM2 interaction by immobilizing biotin-A $\beta$ oligomers on Streptavidin biosensors and then exposing them to sTREM2. These studies showed that sTREM2 associated with A $\beta$ oligomers with two different rates, and dissociated from $A \beta$ oligomers with two different rates (Supplementary Fig. 6iii \& iv). Best fit association and dissociation curves for both wild-type and R47H sTREM2 were with a 2:1 heterogeneous ligand model. In agreement with the semiquantitative experiments described above, these BLI studies confirmed that R47H sTREM2 bound $A \beta$ oligomers less well than wild-type sTREM2 (Supplementary Table 1: TREM2 WT $K_{D} 1$ $=2.00 \pm 0.15 \mu \mathrm{M}, \mathrm{K}_{\mathrm{D}} 2=0.29 \pm 0.08 \mu \mathrm{M}$; TREM2 R47H mutant $\mathrm{K}_{\mathrm{D}} 1=11.70 \pm 5.97 \mu \mathrm{M}, \mathrm{K}_{\mathrm{D}} 2=$ $1.22 \pm 0.30 \mu \mathrm{M}$; where $\mathrm{K}_{\mathrm{D}} 1$ is the dissociation constant of the predominant interaction within the heterogeneous population of $A \beta$ species).

\section{Wild-type sTREM2 inhibits $A \beta$ oligomerisation and dissolves $A \beta$ oligomers, whereas R47H sTREM2 induces $A \beta$ protofibrils}


Because sTREM2 bound to $A \beta$ oligomers, we next investigated whether sTREM2 affected $A \beta$ oligomerization. We incubated $22 \mu \mathrm{M}$ monomeric $A \beta \pm 0.22$ or $0.44 \mu \mathrm{M}$ sTREM2 (wild-type or $\mathrm{R} 47 \mathrm{H}$ ) for 3 hours at $37^{\circ} \mathrm{C}$, i.e. conditions known to generate $A \beta$ oligomers. The formation of $A \beta$ oligomers was then assessed by transmission electron microscopy (TEM) (Fig. 2, Supplemental Fig. 7; dot blotting with A11 anti-oligomer antibody; and by high performance liquid chromatography size exclusion chromatography (HPLC-SEC) experiments (Supplementary Figure 8). These experiments revealed that pre-incubation of $A \beta$ monomers with wild-type sTREM2 (at molar ratios of 1:50 and 1:100 sTREM2: $A \beta$ ) inhibited $A \beta$ oligomerisation (Fig. 2i) quantified by area (Fig. 2ii) or number (Supplementary Fig. 7i). Size exclusion chromatography and antibody dot blots confirmed that sTREM2 inhibited formation of $A \beta$ oligomers (Supplementary Fig. 8i). Analysis of the size distribution of $A \beta$ oligomers by TEM revealed that wild-type sTREM2 reduced the abundance of small oligomers (oligomer size $<60$ $\mathrm{nm}^{2}$ ), and eliminated larger oligomers (oligomer size $>70 \mathrm{~nm}^{2}$ ) (Supplementary Fig. 9i).

In contrast, R47H sTREM2 did not block $A \beta$ oligomerization, but instead promoted the formation of morphologically-distinct $A \beta$ protofibrils (Figure 2iii \& iv \& Supplemental Fig. 7ii \& 9ii). Thus, the presence of wild-type sTREM2 reduces the formation of $A \beta$ oligomers, particularly larger oligomers; and in stark contrast, $\mathrm{R} 47 \mathrm{H}$ sTREM2 induces $A \beta$ monomers to form $A \beta$ protofibrils.

To study reversal of oligomerization, pre-assembled $A \beta$ oligomers were diluted to $2 \mu \mathrm{M}$ and mixed with wild-type or R47H sTREM2 (at molar ratios of sTREM2: total A $\beta$ of: 1:5 and 1:1) and incubated for 30 minutes at $37^{\circ} \mathrm{C}$. The $A \beta$ assemblies were then assessed using the same methods as above. This experiment revealed that wild-type sTREM2 disaggregated pre-formed A $\beta$ oligomers (TEM in Fig. 2iii \& iv \& Supplementary Fig. 7ii; anti-A11 dot blot and HPLC-SEC assays in Supplemental Figures 8ii). Wild-type sTREM2 strongly reduced the abundance of all but the very smallest oligomers (Fig 2iii \& Supplementary Fig. 9ii). In contrast, R47H sTREM2 increased the abundance of $A \beta$ oligomers, and induced the formation of morphologically-distinct A $\beta$ protofibrils (Figure 2iii \& Supplementary Fig. 7ii \& 9ii). A $\beta$ protofibrils are large, linear $A \beta$ oligomers, formed from $A \beta$ monomers in a variety of conditions ${ }^{25,26}$, and are observed in CSF of $\mathrm{MCl}$ and $\mathrm{AD}$ patients, but not healthy controls ${ }^{27}$.

We repeated these experiments at lower concentrations, using preformed $A \beta$ oligomers at $100 \mathrm{nM} \mathrm{A} \beta$ (monomer equivalent) incubated with 20 or $100 \mathrm{nM}$ sTREM2. Wild-type sTREM2 
(at either concentration) induced rapid dissolution of the $A \beta$ oligomers (Supplementary Figs. 10 \& 11). In contrast, treatment with R47H sTREM2 induced aggregation of $A \beta$ oligomers into much larger $A \beta$ assemblies (Supplementary Figs. 10 \& 11).
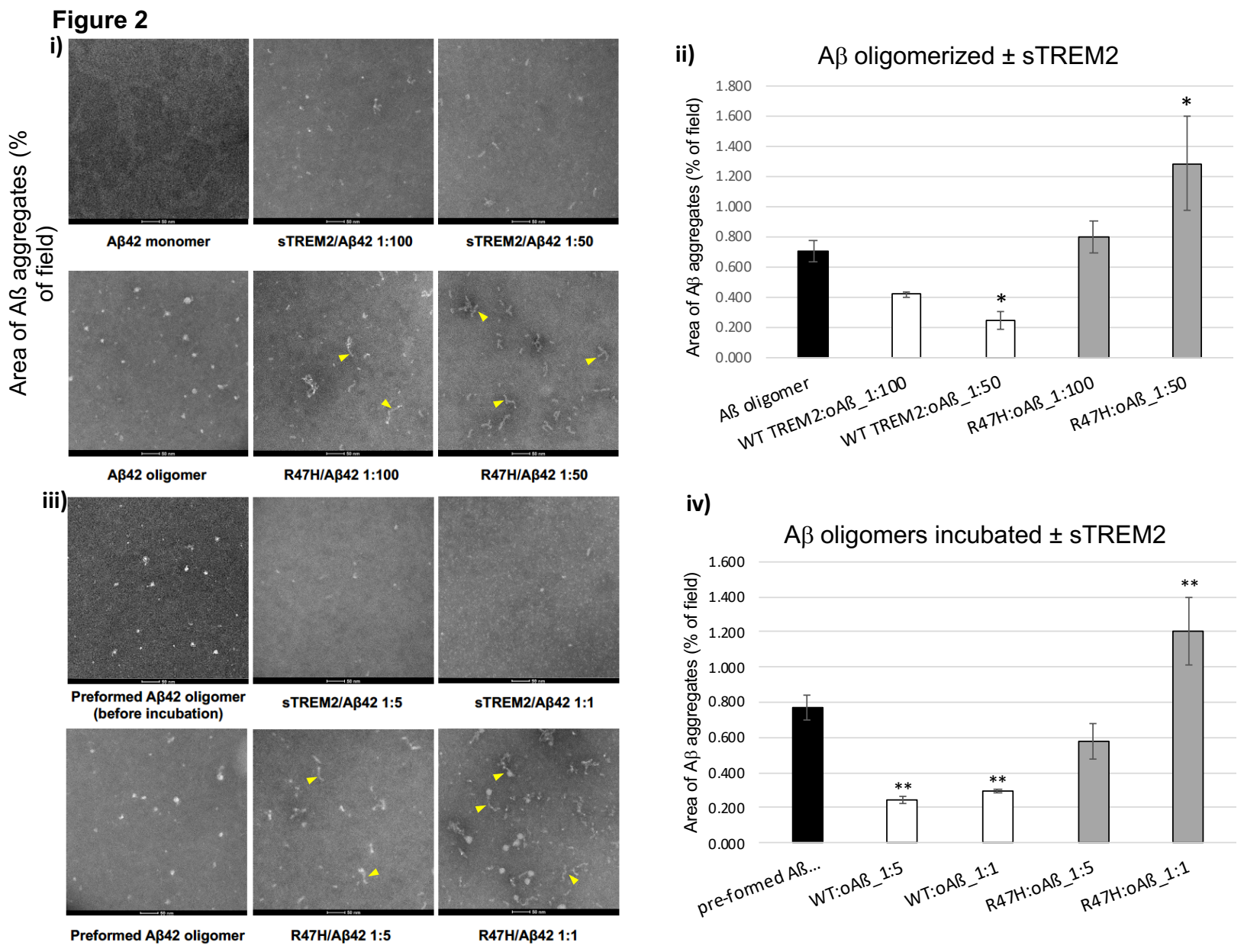

iv)

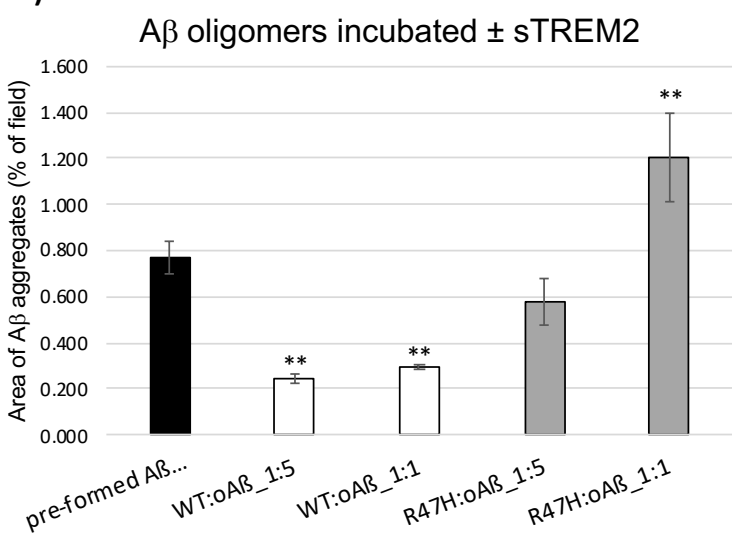

Figure 2. Wild-type sTREM2 inhibits $A \beta$ oligomerization and disaggregates $A \beta$ oligomers, whereas R47H sTREM2 converts $A \beta$ to protofibrils. i) $A \beta$ was aggregated for $3 \mathrm{hr}$ at $37^{\circ} \mathrm{C} \pm$ WT sTREM2 or R47H at molar ratios of 1:100 or 1:50 (sTREM2: A 3 ). TEM images revealed that WT sTREM2 reduced $A \beta$ oligomerization, whereas $R 47 H$ sTREM2 increased proto-fibrils (indicated by yellow arrowheads) and amorphous oligomers. ii) Quantification of three experiments indicated WT sTREM2 decreased and R47H increased area of A $\beta$ aggregates. iii) Preformed $A \beta$ oligomers were treated with WT sTREM2 or R47H at molar ratios of 1:5 or 1:1 for $30 \mathrm{~min}$. TEM images revealed that preformed $A \beta$ oligomers were disaggregated by WT sTREM2, but formed more globular $A \beta$ oligomers and proto-fibrils with R47H sTREM2. iv) Quantification confirmed decreased area of $A \beta$ aggregates with WT sTREM2, and increased 
area with R47H sTREM2. For both ii) \& iv), error bars represent SD, and statistical analysis was performed using one-way ANOVA followed by Bonferroni's multiple comparison test $(n=3$ for each, ${ }^{*} p<0.05$ vs $A \beta$ oligomer, ${ }^{* *} p<0.01$ vs pre-formed $A \beta$ oligomers).

\section{Wild-type sTREM2 inhibited and reversed A $\beta$ fibrillization, whereas $\mathrm{R} 47 \mathrm{H}$ was less effective}

Because wild-type sTREM2 inhibited $A \beta$ oligomerization, and disaggregated $A \beta$ oligomers, we decided to test whether sTREM2 affected $A \beta$ fibrillization. In the absence of sTREM2, $A \beta(10 \mu \mathrm{M})$ fibrillized with standard lag-phase kinetics as measured with Thioflavin $T$ fluorescence. However, $1 \mu \mathrm{M}$ of wild-type sTREM2 almost completely prevented $A \beta$ fibrillization (Fig. 3i-iii). $1 \mu \mathrm{M}$ of R47H sTREM2 did not prevent $A \beta$ fibrillization, but the Thioflavin $T$ fluorescence was lower (Fig. 3i), suggesting fibrils with less $\beta$ sheet structure. Repeating these experiments, at lower concentrations and higher temperature, gave similar results. Thus, fibrillization of $2 \mu \mathrm{M} \mathrm{A} \beta$ was substantially delayed by $20 \mathrm{nM}$ sTREM2 (i.e. a 100:1 ratio), and prevented by $1 \mu \mathrm{M}$ sTREM2 (Fig. 3iv). In contrast, at the same low dose (20nM), R47H sTREM2 had minimal effect on $A \beta$ fibrillization (Fig. 3v).

To test whether sTREM2 could disaggregate fibrils of $A \beta$, small fibrils were preassembled and diluted to $2 \mu \mathrm{M}$ (monomer equivalent) and mixed with wild-type or $\mathrm{R} 47 \mathrm{H}$ STREM2 (at molar ratios of sTREM2: total $A \beta$ of 1:1) and incubated for 30 minutes, 2 hours or 24 hours at $37^{\circ} \mathrm{C}$. Wild-type sTREM2 induced rapid and complete disaggregation of the preformed A $\beta$ fibrils, whereas R47H sTREM2 had little effect on fibrils (Fig. 3vii-ix \& Supplementary Fig. 12), although there may be an increase in oligomers (Fig. 3viii). 


\section{Figure 3}
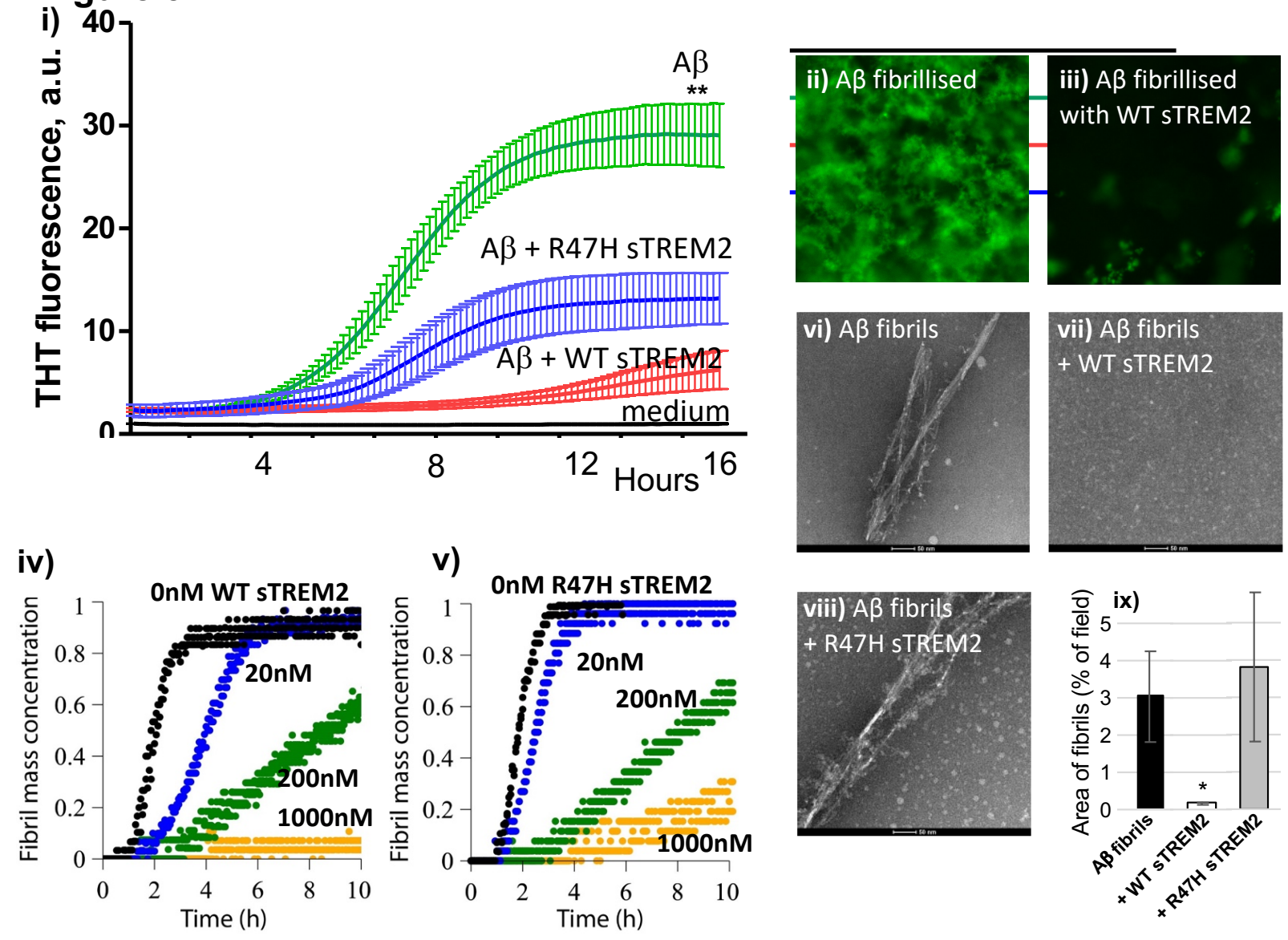

FIGURE 3. Wild-type sTREM2 blocks A $\beta$ fibrillization, but R47H inhibits less.

i) $10 \mu \mathrm{M}$ of monomeric $\mathrm{A} \beta$ was incubated at $30^{\circ} \mathrm{C}$ in DMEM/F12 with $10 \mu \mathrm{M}$ thioflavin T (THT) \pm $1 \mu \mathrm{M}$ wild-type or R47H sTREM2, and the fluorescence followed over time. Means and SD of 3 separate experiments are shown. There was significant difference $\left(p<0.01,{ }^{* *}\right)$ between the final fluorescence of the $A \beta \vee A \beta+W T$ sTREM2 samples. At the end of the assay, ii) in the absence of sTREM2, and iii) presence of wild-type sTREM2, the bottom of the well was imaged using a fluorescence microscope with a x40 objective. iv) $2 \mu \mathrm{M}$ of monomeric $A \beta$ was incubated at $37^{\circ} \mathrm{C}$ in phosphate buffer with $10 \mu \mathrm{M} \mathrm{THT}+0,0.02,0.2$ or $1.0 \mu \mathrm{M}$ wild-type sTREM2, and the fluorescence followed over time. v) $2 \mu \mathrm{M}$ of monomeric $A \beta$ was incubated at $37^{\circ} \mathrm{C}$ in phosphate buffer with $10 \mu \mathrm{M}$ THT $+0,0.02,0.2$ or $1.0 \mu \mathrm{M}$ R47H sTREM2, and the fluorescence followed over time. Preformed $A \beta$ fibrils were either vi) untreated, vii) treated with WT sTREM2, or viii) treated with R47H sTREM2 at molar ratios of $1: 1$ and TEM imaged after 30min. ix) Quantification of the area of $A \beta$ fibrils confirmed that WT sTREM2 decrease $A \beta$ fibrils, and R47H sTREM2 had no effect. Error bars represent SD. Statistical analysis was performed using 
one-way ANOVA followed by Bonferroni's multiple comparison test ( $n=3-8,{ }^{*} p<0.05$ vs preformed $A \beta$ fibril).

\section{Wild-type sTREM2 inhibits, while R47H sTREM2 increases $A \beta$ oligomer neurotoxicity}

Because sTREM2 bound $A \beta$ oligomers and inhibited $A \beta$ oligomerisation and fibrillization, we next tested whether sTREM2 affected the neurotoxicity of $A \beta . A \beta$ neurotoxicity is thought to be mediated by 2 different mechanisms - namely by permeabilization of neuronal membranes ${ }^{28}$, and by microglial activation ${ }^{28,29}$.

Consequently, we initially used nanosized phospholipid membrane vesicles containing a calcium-sensitive fluorophore to explore the ability of $A \beta$ to permeabilize membranes. Prior work has shown that $A \beta$ oligomers (but not monomers or fibrils) can permeabilize these membranes 28. We found that wild-type sTREM2 and R47H sTREM2 proteins themselves had no effect on membrane permeabilization when added in the absence of $A \beta$ (Supplementary Fig. 13i). By contrast, $A \beta$ oligomerized for 6 hours in the absence of sTREM2 induced permeabilization of the vesicles. However, $A \beta$ oligomerized in the presence of wild-type sTREM2 (at molar ratios of STREM2: total $A \beta$ of $1: 10$ ) induced significantly less permeabilization (Figure 4i). Wild-type sTREM2 also inhibited the permeabilization induced by pre-formed $A \beta$ oligomers (at a 1:1 molar ratio) (Fig. 4ii). The simplest explanation for this inhibition of permeabilization is our previous finding that wild-type sTREM2 inhibits $A \beta$ oligomerization and disaggregates $A \beta$ oligomers at these concentrations. However, we do not discount the possibility that binding of wild-type sTREM2 to $A \beta$ oligomers might also reduce their toxicity.

When these experiments were repeated using R47H sTREM2 (at a molar ratio of sTREM2: total $A \beta$ of $1: 10$ ) we found that $A \beta$ oligomers formed in the presence of $R 47 H$ sTREM2 also induced less permeabilization than $A \beta$ oligomerized alone, but allowed more permeabilization than $A \beta$ oligomerized with wild-type sTREM2 (Fig. 4i). In contrast to wild-type sTREM2, R47H sTREM2 did not significantly inhibit permeabilization induced by pre-formed $A \beta$ oligomers (Figure 4ii). These results are consistent with wild-type sTREM2 reducing A $\beta$ oligomer abundance, but R47H sTREM2 not reducing $A \beta$ oligomer abundance (Fig. 2).

We next tested whether sTREM2 could block the neurotoxicity induced by $A \beta$ in neuronal-glial co-cultures. We have previously shown that $250 \mathrm{nM} \mathrm{A} \beta$ induces slow, progressive neuronal loss mediated by microglia in this co-culture system ${ }^{29}$. We found that $250 \mathrm{nM} \mathrm{A} \beta$ 
induced neuronal loss over three days, and this $A \beta$-induced neuronal loss was substantially reduced by co-treatment with $25 \mathrm{nM}$ wild-type sTREM2 (Fig. 4iii \& iv). In contrast, co-treatment of the cultures with $25 \mathrm{nM}$ R47H sTREM2 increased the neuronal loss above the level induced by $250 \mathrm{nM} \mathrm{A} \beta$ alone (Fig. 4iv). Wild-type and R47H sTREM2 proteins by themselves had no significant effect on neuronal loss (Supplementary Fig. 13ii). As membrane permeabilization is thought to be mediated by smaller $A \beta$ oligomers, while microglial activation is thought to be mediated by larger $A \beta$ aggregates ${ }^{28}$, our finding that R47H sTREM2 increased $A \beta$-induced neuronal loss (Fig. 3iv), which is known to be microglia-mediated ${ }^{29}$, is consistent with R47H sTREM2 increasing larger $A \beta$ aggregates (Fig. 2). Overall, our results indicate that wild-type sTREM2 reduces $A \beta$ neurotoxicity, while $R 47 H$ TREM2 increases $A \beta$ neurotoxicity.

\section{Figure 4}
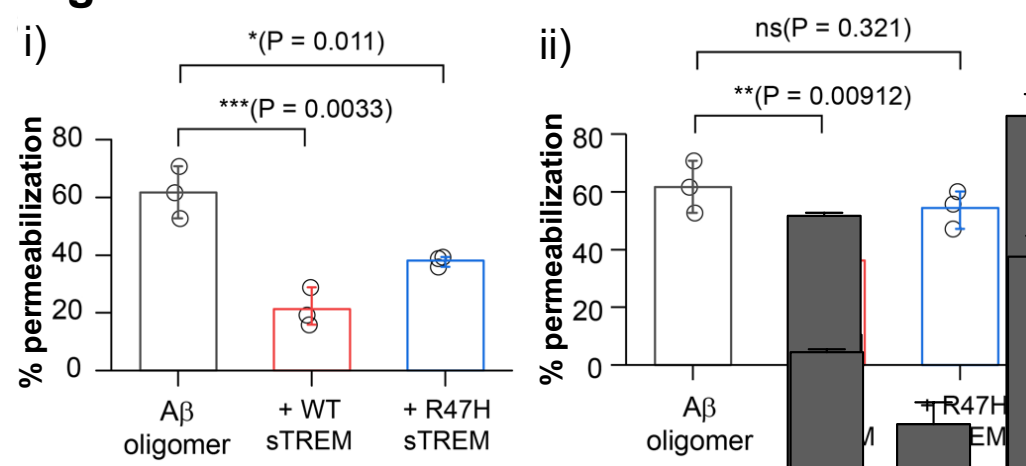

iii)
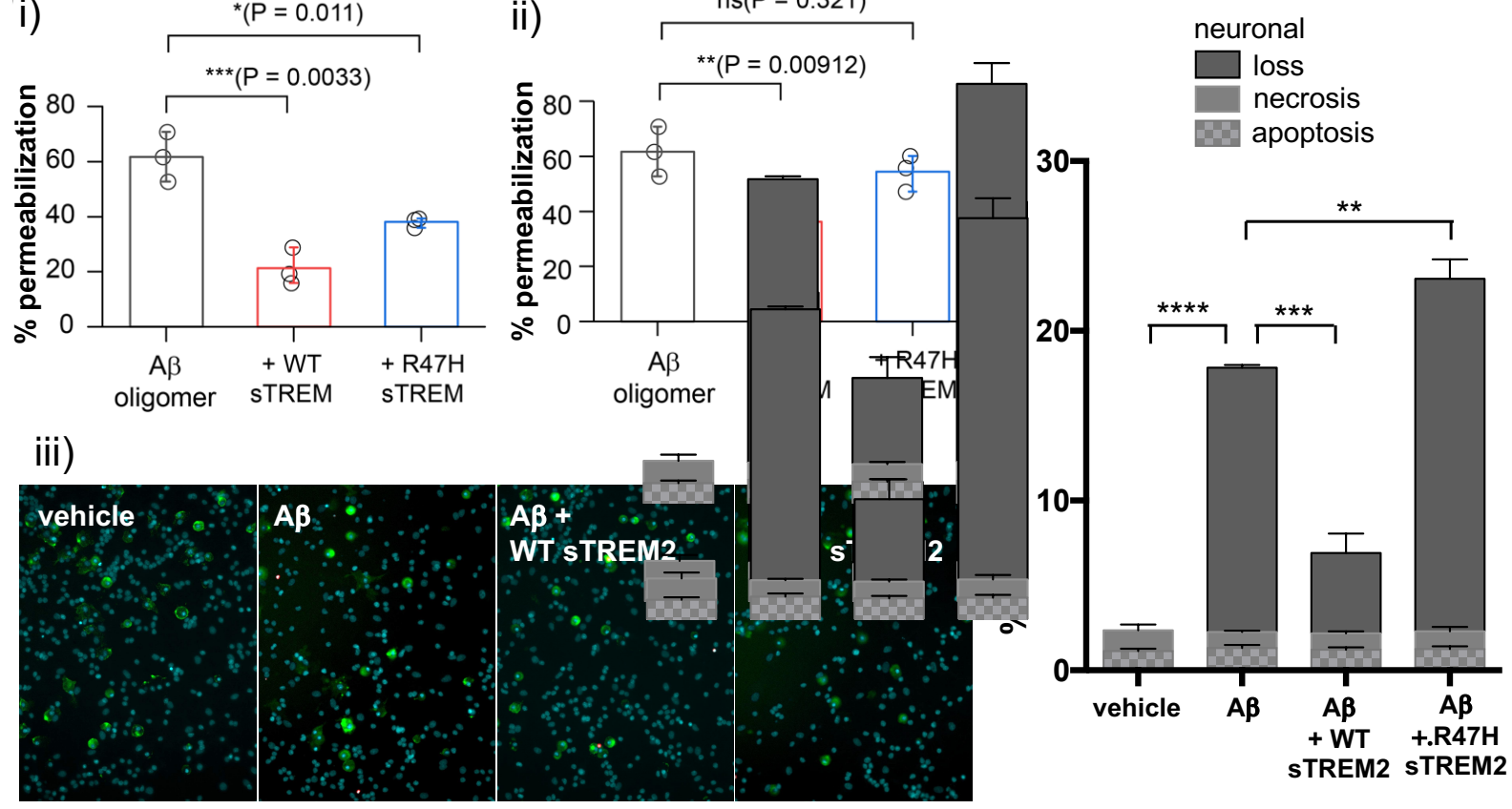

FIGURE 4. Wild-type sTREM2 blocks $A \beta$-induced membrane permeabilization and neuronal loss, but R47H sTREM2 inhibits less. i) $10 \mu \mathrm{M}$ of monomeric $A \beta$ was aggregated \pm $1 \mu \mathrm{M}$ wild-type (WT) or R47H sTREM2 for 6 hours, diluted to $200 \mathrm{nM}$ and membrane permeabilization assay was performed. Error bars = SD of three independent experiments; ${ }^{* *}=p<0.01$; statistics: two sample t-test. $\quad$ ii) $10 \mu \mathrm{M}$ of monomeric $A \beta$ was aggregated for 6 hours, diluted to $200 \mathrm{nM}$, then incubated for 30 minutes with vehicle, $200 \mathrm{nM}$ WT sTREM2 or R47H sTREM2, before the membrane permeabilization assay was performed. Error bars $=S D$ 
of three independent experiments; ${ }^{* *}=p<0.01$; statistics: two sample t-test. iii $\&$ iv) Mixed neuronal-glial co-cultures were treated with either: vehicle, $250 \mathrm{nM}$ monomeric $A \beta, 250 \mathrm{nM} A \beta+$ 25 nM WT sTREM2 or 250 nM A + 25 nM R47H sTREM2. Three days later, cultures were stained with isolectin B4 (green, to identify microglia), propidium iodide (red, to identify dead cells) and Hoechst 33342 (blue, to identify cells and whether apoptotic) and imaged (representative fields: iii), and the number of apoptotic, necrotic and healthy neurons were quantified (mean data: iv). Loss is the decrease in neuronal density relative to vehicle treated cultures. Error bars = SEM; ${ }^{* *}=p<0.01,{ }^{* * *}=p<0.001,{ }^{* * *}=p<0.0001 ; n=4$ independent experiments on separate cell cultures. Statistical analysis was by one-way ANOVA and Tukey's post hoc test.

\section{Discussion}

We have shown that $A \beta$ oligomers, but not $A \beta$ monomers or fibrils, bind to microglial TREM2 and induce sTREM2 release, and this released STREM2 also preferentially binds to A $\beta$ oligomers, consistent with previous reports ${ }^{13-15}$. Crucially however, we also found that wild-type sTREM2 inhibits the formation of fibrils and larger $A \beta$ oligomers and disaggregates protofibrils and larger $A \beta$ oligomers into the smallest $A \beta$ oligomers. In contrast, R47H sTREM2 promoted the formation of $A \beta$ protofibrils, indicating a gain of function by this mutation. These effects may provide a partial explanation of how a single copy of the TREM2 R47H mutant is associated with increased risk for $A D$, by increasing production of neurotoxic forms of $A \beta$.

One potential caveat is the protein concentrations used in our experiments. In order to observe $A \beta$ aggregation over a reasonable experimental timeframe, the lowest concentration of $A \beta$ used here was $100 \mathrm{nM}$, whereas levels found in CSF are about $0.1 \mathrm{nM}^{10,20,30}$. However, the $A \beta$ oligomer concentrations near amyloid plaques was estimated as $700 \mathrm{nM}^{31}$, and plagues are likely be a more relevant location for $A \beta$ aggregation in $A D$ than CSF. STREM2 concentrations in lumbar CSF average about $0.2 \mathrm{nM}^{10,20,30}$; however again, it is likely that sTREM2 levels are higher near amyloid plaques surrounded by activated microglia, which express high levels of TREM $^{3}$. Note also that the observed effects of sTREM2 on A $\beta$ are rapid: 20 nM sTREM2 dissolved $100 \mathrm{nM}$ A $\beta$ oligomers within 30 mins in vitro (Supplementary Fig 11). Lower concentrations are likely to have the same disaggregating effect over a slower time course, which may be more relevant to the slow time course of $A D$. 
We did not investigate the molecular mechanisms by which sTREM2 affected $A \beta$ aggregation, but our finding that wildtype sTREM2 stabilises the smallest observable oligomers (Supplementary Figure $9 \& 11$ ), suggests the possibility that sTREM2 binds preferentially to these (or undetectable oligomers such as dimers), thereby potentially blocking further growth of aggregates, and disaggregating larger $A \beta$ aggregates into smaller, potentially non-toxic forms. Precedent for this is the finding that $\alpha$-synuclein-specific single-domain antibodies (nanobodies) bind $\alpha$-synuclein stable oligomers and convert them into less stable oligomers with reduced toxicity $^{32}$. However, clearly more research is required to determine the mechanisms for sTREM2 and $A \beta$.

In summary, our experimental data reveal how $A \beta$ oligomer binding to TREM2 may mediate direct (via activation of intracellular TREM2 dependent signalling pathways) and indirect protective mechanisms (via effects on $A \beta$ oligomer assembly and toxicity). Our studies are broadly congruent with previous research showing that knockout of TREM2 expression in APP mice, resulted in accelerated amyloid plaque seeding ${ }^{17}$, with increased protofibrillar halos and hotspots around these plaques ${ }^{3,16,18}$. Our studies provide a potential explanation of the clinical observation of slower rates of cognitive and clinical decline in patients with $\mathrm{MCl}$ or $\mathrm{AD}$ who have higher levels of STREM2 in the $\mathrm{CSF}^{20,21}$, and slower rates of amyloid deposition in healthy controls and $\mathrm{MCl}$ patients with higher STREM2 levels in $\mathrm{CSF}^{22}$. They also provide a potential explanation for the beneficial effects of infusing or expressing STREM2 into the brain of mouse models of $A D^{16}$. Additional biophysical studies will be required to identify the key $A \beta$ species dynamically interacting with wild-type sTREM2 to prevent neurotoxicity, and the A $\beta$ species stabilised by sTREM2 R47H to increase neurotoxicity. This knowledge could be exploited for the design of small brain-penetrant molecular mimics of sTREM2 as potential therapeutics for AD.

\section{METHODS}

\section{cDNA constructs}

Wild-type human TREM2 (hTREM2) and DAP12 (hDAP12) were subcloned in pCMV6-A vector (Origene, Rockville, MD, USA). A single $\mathrm{C} \rightarrow$ A nucleotide polymorphism (SNP) at codon 85 (T85K) on the Origene TREM2 cDNA clone was reverted to consensus wild-type sequence by site-directed mutagenesis.

\section{Antibodies}


The following antibodies were used: human TREM2 (\#AF1828, 1:100 for immunocytochemistry (ICC); 1:1000 for western blot; R\&D); mouse TREM2 (\#AF1729, 1:500 for western blot; R\&D); DDK (FLAG) (\#TA50011, 1:2000 for western blot; Origene); V5 (\#46-0705, 1:2000 for western blot; Life Technologies, Burlington); Nicastrin (\#sc-14369, 1:200 for western blot; Santa Cruz); GAPDH (\#2118, 1:3000 for western blot; Cell Signaling); Anti-His (C-term)HRP (\#46-0707, 1:2000 for dot blot, Invitrogen); A $\beta$ (6E10, 1:1000 for western blot and dot blot, Covance); TrueBlot secondary antibodies conjugated with horseradish peroxidase (anti-goat $\lg$, anti-mouse IgG and anti-sheep lgG, 1:1000 for western blot after immunoprecipitation; Rockland); Secondary antibodies conjugated with horseradish peroxidase (anti-mouse IgG; antirabbit IgG, 1:2000 for western blot; Thermo Scientific). Function-blocking TREM2 antibody (Clone 78.18, $25 \mu \mathrm{g} / \mathrm{ml}$ for blocking assay, AbD Serotec catalogue number MCA4772EL) and a control antibody (Rat IgG1, AbD Serotec) were used for blocking experiments.

\section{Animals}

All experiments were performed in accordance with Canadian Council on Animal Care guidelines and UK Animals (Scientific Procedures) Act (1986) and approved by the Animal Care Committee at the University of Toronto and the Cambridge University local ethics committee.

TREM2 deficient mice were obtained from Dr. J. Gommerman, and were constructed by targeted homologous recombination ${ }^{33}$, which removed Exon 1 and 2, which include the start codon and the major extracellular IgG domain. In contrast to the recently reported Velocigene construct, the direction of the Hygromycin cassette was "reversed". Crucially, in agreement with two other models, but in contrast to the Velocigene construct, RT-PCR analyses confirmed specific loss of TREM2 expression without the perturbation of TREM1L expression observed in the Velocigene construct ${ }^{33}$.

\section{Primary microglial cell culture}

Primary cultures of mixed glial cells and pure microglia were prepared from the cerebral cortex of P0-3 day old C57BL/6 TREM2 ${ }^{+/+}$or TREM2/-- mice as described ${ }^{33}$. After removal of olfactory bulbs, cerebral hemispheres were cut into $\sim 1 \mathrm{~mm}$ pieces, vortexed for 1 minute, filtered through a $40 \mu \mathrm{m}$ cell strainer and plated in $80 \mathrm{~cm}^{2}$ tissue culture flasks. Mixed glia were cultured until microglia appeared (10-18 days after plating). Microglia were isolated by shaking flasks. Purified microglia were replated for analysis. On average, each experiment used microglia from 
30-35 pups. All pups used in this study (wild type; $\mathrm{R} 47 \mathrm{H}$, and TREM2KO) were maintained on a C57BL/6 background.

\section{Mixed neuronal-glial co-cultures and treatments}

Mixed neuronal-glial co-cultures were prepared from cerebella of 5-7 day-old rats ${ }^{33}$, cultured for 7 days, then treated with either: vehicle, $250 \mathrm{nM}$ monomeric amyloid beta 1-42 (A $\beta)$, $250 \mathrm{nM} \mathrm{A} \beta+25 \mathrm{nM}$ wild-type sTREM2 or $250 \mathrm{nM}$ A $\beta+25 \mathrm{nM}$ R47H sTREM2 for 3 days. Three days later, neuronal death and loss was quantified by staining the live cultures with propidium iodide for necrosis, with isolectin IB4 for microglia, and with Hoechst 33342 for nuclei, which is used to distinguish healthy (uncondensed) from apoptotic (condensed) nuclei, and to distinguish astrocytes (large bean-shaped nuclei) from neurons and microglia (small, round nuclei). The number of apoptotic, necrotic and healthy neurons, astrocytes and microglia are then counted on photos of multiple set fields, as previously described ${ }^{29,33}$,

\section{Amyloid $\beta$-peptide}

Human A $\beta 42$ (used in TREM2 cleavage), HiLyte Fluor 488-labelled A 342 (used in Single molecule TIRF imaging), HiLyte Fluor 647-labelled Aß42 (used in IP and dot blot) and Biotin$A \beta 42$ (used in BLI) were purchased from AnaSpec and Bachem; A 42 oligomers were prepared as endotoxin-free preparations ${ }^{34}$, and validated by antibodies, gels and electron microscopy (Supplimentary Figure 1A). Prior work has established that these fluorescent tags do not significantly impact $A \beta$ assembly ${ }^{35}$.

\section{Recombinant ectodomain expression and purification}

cDNAs encoding residues 19-143 of WT and R47H TREM2 were ordered as linear DNA strings (Life Technologies) and inserted into pHLSec. The plasmids were transfected into Freestyle 293-F cells (Life Technologies) grown in suspension in FreeStyle293 medium. Cells were transfected at a density of $2.5 \times 10^{6} / \mathrm{ml}$ with a DNA concentration of $3 \mu \mathrm{g} / \mathrm{ml}$ and polyethyleneimine (Linear PEI 25 kDa molecular weight, PolySciences Inc) at $9 \mu \mathrm{g} / \mathrm{ml}$. On day 6 post-transfection, conditioned medium was harvested and TREM2 protein was purified using IMAC (Histrap excel, GE Healthcare) followed by gel filtration chromatography in storage buffer - 20 mM HEPES, 200 mM NaCl, pH 7.0. To maintain a low endotoxin level: endotoxin-free chemicals and plasticware were used; all chromatography media, columns and concentrators (Vivaspin Turbo) were soaked for $>12$ hours in $1 \mathrm{M} \mathrm{NaOH}$. Proteins in storage buffer were concentrated to between $5-10 \mathrm{mg} / \mathrm{ml}$ for use and tested for endotoxin with the EndoZyme 
recombinant Factor $\mathrm{C}$ assay $(\mathrm{Hyglos} \mathrm{GmbH})$. Endotoxin levels in all assays were maintained at $<0.1 \mathrm{EU} / \mathrm{ml}$.

\section{Co-immunoprecipitation and western blot}

Primary microglia were rinsed with ice-cold DMEM without phenol red and incubated with $100 \mathrm{nM}$ HiLyte Fluor 647 -labelled $\mathrm{A} \beta$ at $4^{\circ} \mathrm{C}$ for 1 hour. For antibody blocking assays, before $A \beta$ incubation, microglia cultures were pre-incubated with $25 \mu \mathrm{g} / \mathrm{ml}$ monoclonal antiTREM2 antibody or rat lgG1 control for 20 minutes Cells were collected by scraping, washed with ice cold 1xPBS and centrifuged. Primary microglia were lysed with 1\% CHAPSO buffer (1\% 3-[(3-cholamydopropyl) dimethylammonio]-2-hydroxy-1-propanesulfonate (CHAPSO), 25 mM HEPES, 150 mM NaCl, 2 mM EDTA, pH 7.4). Crude membrane fractions were extracted from HeLa cell pellets as described ${ }^{36}$ and lysed with $1 \%$ CHAPSO buffer. Primary antibodies and corresponding $\operatorname{lgG}$ controls ( $1 \mu \mathrm{g} /$ reaction) were incubated with lysates at $4^{\circ} \mathrm{C}$ for 2 hours, then mixed with Protein G Dynabeads (Life Technologies), incubated at $23^{\circ} \mathrm{C}$ for 20 minutes, then washed and eluted as per manufacturer's instructions. The immunoprecipitates were separated on 4-12\% MES/Bis-Tris gels (Life Technologies). Fluorescent signals of $A \beta$ were detected using an Odyssey FC imaging system (LI-COR). Proteins were transferred onto nitrocellulose membranes, which were probed with primary antibodies and corresponding TrueBlot secondary antibodies.

In vitro co-precipitation of TREM2 or TREM1 with $A \beta$ oligomers was performed by mixing recombinant Fc-tagged TREM2 or TREM1 ectodomain (residues 19-171; 100 ng/ml, R\&D systems), 100 nM HiLyte Fluor 647-labelled A oligomers and Protein G Dynabeads in 0.5\% fatty acid free BSA PBS-Tween buffer. The mixture was incubated at room temperature for 1 hour and then washed, eluted and analysed the same as described above.

\section{Dot blot}

$A \beta$ monomers or oligomers $(1 \mu \mathrm{g} / \mathrm{dot})$ prepared as described above and anti-TREM2 antibody $(0.3 \mu \mathrm{g} / \mathrm{dot})$ were spotted onto a nitrocellulose membrane. Membrane strips were blocked with $3 \%$ fatty acid free BSA (Sigma Aldrich) and then incubated with $100 \mathrm{nM}$ recombinant His-tagged WT/R47H TREM2 ECD diluted in blocking buffer at $4^{\circ} \mathrm{C}$ for 1 hour. Bound TREM2 ECDs were probed with HRP-conjugated anti-His tag antibody and detected with Odyssey FC imaging system. 


\section{Bio-Layer Interferometory}

i) Preparation of $A \boldsymbol{\beta}$ oligomers for Bio-Layer Interferometry (BLI) studies. Synthetic human $A \beta$ residues 1-42 and biotinylated $A \beta 42$ were purchased from Bachem or AnaSpec and solubilized in hexafluoroisopropanol (HFIP) with DMSO $(200 \mu \mathrm{l})$ to generate monomeric species. Solubilized $A \beta$ was diluted in filtered assay buffer $[20 \mathrm{mM} \mathrm{HEPES}, 500 \mathrm{mM} \mathrm{NaCl}$ $\mathrm{pH} 7.4$ ] containing DMSO (2\%) to a total volume of $9800 \mu \mathrm{l}$ and a peptide concentration of 0.1 $\mathrm{mg} / \mathrm{ml}(22 \mu \mathrm{M})$. Peptide solutions were aliquoted and incubated for 3 hours at $37^{\circ} \mathrm{C}$ with stock solutions stored at $-80^{\circ} \mathrm{C}$. Transmission electron microscopy (TEM) was performed using undiluted biotinylated $A \beta(100 \%)$ or a mixture of biotinylated $A \beta(10 \%)$ and unlabelled $A \beta(90 \%)$ which were diluted to final concentrations of $1.1 \mu \mathrm{M}$ (Supplementary Figure 1iii \& iv). These TEM samples $(10 \mu \mathrm{l})$ were applied to carbonate coated grids and negatively stained with $1 \%$ phosphotungstic acid (PTA). TEM micrographs indicating the globular morphology of the A $\beta$ oligomers were obtained on a Hitachi $\mathrm{H}-7000$ operated at $75 \mathrm{kV}$. The biotinylated oligomers were indistinguishable from non-biotinylated $A \beta$ oligomers.

\section{ii) Binding affinity of TREM2 ectodomain to $A \beta$ was analyzed by Bio-Layer Interferometry} (BLI) using the Octet RED384 system (ForteBio). Assays were performed at $30^{\circ} \mathrm{C}$ in the assay buffer (20 mM HEPES, $500 \mathrm{mM} \mathrm{NaCl}, 0.1 \%$ w/v BSA, 0.02\% v/v Tween20, pH 7.4) with vibration at 1,000 rpm. Streptavidin biosensors loaded with Biotin-A $\beta$ (Anaspec) were incubated with TREM2 ectodomain WT $(1,2,5,10 \mu \mathrm{M})$ or $\mathrm{R} 47 \mathrm{H}(2,5,10,20 \mu \mathrm{M})$ to obtain the association curves, and subsequently in the assay buffer to obtain the dissociation curves. Kinetics data were analyzed using a 2:1 heterogeneous ligand model.

\section{Single molecule TIRF imaging}

i) Aggregation of $A \beta$ for TIRF studies. Stock solutions of HiLyte ${ }^{\text {TM }}$ Fluor 488- labeled Human A 342 ( $A \beta, 0.1 \mathrm{mg}$; AnaSpec, USA) were prepared by dissolving the lyophilized peptide (1\% $\mathrm{NH}_{4} \mathrm{OH}, 50 \mu \mathrm{l}$ ) followed by dilution into $\mathrm{pH} 7.4 \mathrm{PBS}$ to $200 \mu \mathrm{M}$, aliquoted and stored at $-80^{\circ} \mathrm{C}$. Working solutions were prepared by diluting the monomeric stock solutions into $\mathrm{pH} 7.4 \mathrm{PBS}$ on ice to the concentration used for the aggregation $(0.5 \mu \mathrm{M})$. The working solution was then placed into a shaking incubator $\left(3^{\circ} \mathrm{C}, 200 \mathrm{rpm}\right)$ for 6 hours to ensure a significant population of oligomeric species. All protein samples were stored and diluted into LoBind microcentrifuge tubes (Eppendorf, Hamburg, Germany). 
ii) Preparation of slides for TIRF microscopy. Slides were prepared in the same way as previously described ${ }^{23}$. Slides containing unlabelled $A \beta$ and TREM2 were tested for fluorescence artefacts.

iii) TIRF Microscope set-up. Co-localization experiments were performed on a bespoke TIRF set-up. Continuous wave solid-state lasers operating at $488 \mathrm{~nm}$ and $641 \mathrm{~nm}$ were used for imaging. The beam power was controlled by attenuation through a neutral density filter, following which undesirable wavelengths were filtered out through excitation filters (LL01-48825, FF01-640/14-25, Semrock). The beams were then circularly polarised by a quarter wave plate. Laser lines were combined by dichroic mirrors. The beams were then passed through a Köhler lens into the back aperture of an inverted microscope body (Olympus, IX73) and were reflected by a dichroic mirror (Di01-405/488/532/635, Semrock) through an oil immersion objective (Olympus, 60XOTIRF). Emitted radiation was collected through the same objective and passed through the dichroic before being filtered (FF01-480/40-25, LP02-647RU-25, Semrock). The fluorescence emission was then projected onto an EMCCD camera (Evolve Delta, Photometrics), each pixel was $275 \mathrm{~nm}$ in length. Data visualisation achieved through Micro-Manager and ImageJ software.

iv) Single-molecule TIRF Imaging. The co-localization experiment was designed to investigate binding of $A \beta$ oligomers with TREM2. The $A \beta 6$ hour aggregation contained a mixture of monomeric and oligomeric species hence illumination was performed for significant time periods to photo-bleach the monomeric species. Automated TIRF co-localization experiments were performed with the use of a custom script (BeanShell, micromanager). Each data set consisted of a $3 \times 3$ grid of 9 images at different areas of the coverslide, distances between images was $350 \mu \mathrm{m}$ as previously described ${ }^{20}$. Images were recorded at $33 \mathrm{~ms}$ exposure, beginning with 800 frames excitation with $641 \mathrm{~nm}$ followed by 800 frames excitation $488 \mathrm{~nm}$ excitation in the same field of view.

v) Co-localization Analysis. Co-localization data was analyzed with a bespoke ImageJ macro. The $488 \mathrm{~nm}$ illumination channel contained a mixture of monomeric and oligomeric $A \beta$ species, it was determined that the majority of monomeric species were photo-bleached by 40 frames of $488 \mathrm{~nm}$ illumination. Therefore, only frames after this point were considered in this analysis. The $488 \mathrm{~nm}$ and $641 \mathrm{~nm}$ illumination channels were compressed in time to create two single frame images representing the average pixel intensities. Following which, points of intensity 
representing HiLyte ${ }^{\mathrm{TM}}$ Fluor $488 \mathrm{~A} \beta$ or AF647-TREM2 above a background threshold were located, counted and binary images of these maxima were created. The two images were then summed to identify co-localized points. Chance co-incident spots were excluded by $90^{\circ}$ rotation of the binary image representing AF647-TREM2 points and summation with the HiLyte ${ }^{\text {TM }}$ Fluor $488 \mathrm{~A} \beta$ image. Chance coincident spots were subtracted from the actual coincidence value and percentage coincidence was calculated with the equation below:

$$
\text { Coincidence }=\left(\frac{N_{A \beta-T R E M 2}}{\left(N_{A \beta-T R E M 2}-N_{A \beta}\right)}\right) \times 100
$$

\section{TREM2 cleavage assays}

Primary microglia from wild and TREM2 mutant mice were isolated from mixed glia culture by shaking and grown on 96 -wells plates for 24 hours. Media were replaced by treatment media ( $1 \mathrm{ml}$ of OptiMEM supplemented with $0.5 \%$ FBS) containing defined concentrations of freshly made $A \beta$ oligomers. Cells were collected 24 hours after treatment and NTF release in conditioned media and TREM2 in cell lysate were detected by ELISA. TREM2 ELISA was performed on 96-well plates previously coated by TREM2 antibody (clone 78.18) and blocked with $0.5 \%$ BSA in $0.05 \%$ Tween20 in 1x PBS (PBST). Medium or cell lysate samples were added to pre-coated wells and incubated overnight at $4^{\circ} \mathrm{C}$. After washing with PBST, wells were incubated with TREM2 antibody (sheep anti-mouse TREM2, AF1729) diluted in blocking buffer for 2 hours at room temperature. After secondary antibody (anti-sheep-HRP, 1:5000) incubation for 1 hour at room temperature and washing, TMB chromogenic substrate was added to each well and developed at room temperature in dark for 20 minutes. The absorbance at 450nm was read immediately after stop solution was added. TREM2 cleavage was represented by the ratio of relative values of STREM2 in medium and total TREM2 in cell lysate, after subtracting the corresponding background values from TREM2 KO microglia in the same ELISA experiment.

\section{sTREM2 effects on $A \beta$ oligomers}

i) Preparation of $A \beta$ oligomers and $A \beta$ fibrils and sTREM2 treatment. HFIP-treated human synthetic $A \beta 42$ were purchased from Bachem. $A \beta$ solubilized by DMSO was diluted in PBS $(\mathrm{pH}$ $7.4)$ to a peptide concentration of $0.1 \mathrm{mg} / \mathrm{mL}(22 \mu \mathrm{M})$. To study sTREM2 effects on assembly of $A \beta$ oligomers, monomeric $A \beta(22 \mu \mathrm{M})$ solutions were incubated with or without WT sTREM2 or sTREM2 R47H (sTREM2: $A \beta, 1: 100$ or $1: 50$ ) for 3 hours at $37^{\circ} \mathrm{C}$. To study reversal of oligomerisation, monomeric $A \beta(22 \mu \mathrm{M})$ solutions were incubated for 3 hours at $37^{\circ} \mathrm{C}$ to make 
oligomers then diluted and mixed with WT sTREM or sTREM2 R47H (sTREM2: Aß, 1:5 or 1:1) to a $A \beta$ concentration of $2.2 \mu \mathrm{M}$. Then $A \beta$ and sTREM2 mixture were incubated for 30 minutes at $37^{\circ} \mathrm{C}$. To study dissociation of fibrils, monomeric $A \beta(110 \mu \mathrm{M})$ solutions were incubated for 24 hours at $37^{\circ} \mathrm{C}$ with shaking (150 rpm) then diluted and mixed with WT or R47H sTREM2 (sTREM2: $A \beta, 1: 1$ ) to a $A \beta$ concentration of $2.2 \mu \mathrm{M}$, then incubated for 30 minutes, 2 hours or 24 hours at $37^{\circ} \mathrm{C}$. These peptide solutions were then used for TEM, dot-blot and HPLC-SEC experiments.

ii) Transmission electron microscopy (TEM) $A \beta$ oligomers were prepared as described above. Transmission electron microscopy (TEM) was performed to characterize $A \beta$ or mixtures of sTREM2 and $A \beta$ which were diluted to final $A ß$ concentrations of $1.1 \mu \mathrm{M}$ or $100 \mathrm{nM}$. These TEM samples $(10 \mu \mathrm{L})$ were applied to carbonate coated grids for $1 \mathrm{~min}$ and negatively stained with $1 \%$ phosphotungstic acid (PTA) for $1 \mathrm{~min}$. TEM micrographs were obtained on a Hitachi $\mathrm{H}$ 7000 operated at $120 \mathrm{kV}$.

iii) Dot blots. $A \beta$ oligomers were prepared as described above. $A \beta$ and mixtures of sTREM2 and $A \beta$ oligomers $(1 \mu \mathrm{g} / \mathrm{dot})$ prepared as described above were spotted onto a nitrocellulose membrane. Membrane strips were blocked with $5 \%$ fatty acid free BSA (Sigma Aldrich) and then incubated with anti-oligomer specific antibody (A11, ThermoFisher Scientific) or antiamyloid $\beta$ sequence specific antibody (4G8, Millipore) for $1 \mathrm{hr}$ at RT and probed with HRPconjugated anti-rabbit or mouse lgG antibodies and detected with ChemiDoc XRS+ imaging system (Bio-Rad).

iv) HPLC-SEC. A $\beta$ oligomers were prepared as described above. The size of prepared A $\beta$ peptides were analyzed by size exclusion chromatography (SEC). Samples were run on a BioSep SEC-S 4000 (Phenomenex) column using a ProStar 210 HPLC (Varian) system with a ProStar 325 UV detector (Varian). Injected samples were eluted with PBS (pH 7.4) at a flow rate of $0.5 \mathrm{~mL} / \mathrm{min}$ at ambient temperature, and data were obtained at $280 \mathrm{~nm}$. Peak areas were integrated using Star 6.2 Chromatography Workstation (Varian).

\section{A $\beta$ fibrillization assay}

$10 \mu \mathrm{M}$ of monomeric $\mathrm{A} \beta$ was incubated at $30^{\circ} \mathrm{C}$ in DMEM/F12 with $10 \mu \mathrm{M}$ Thioflavin $\mathrm{T} \pm 1 \mu \mathrm{M}$ wild-type or R47H sTREM2 in Corning® 96 Well White Polystyrene Microplate with a clear flat bottom in a FluoroSTAR Optima plate reader (with orbital shaking between readings) to monitor 
fluorescence. Alternatively, $2 \mu \mathrm{M}$ of monomeric $A \beta$ was incubated at $37^{\circ} \mathrm{C}$ in $20 \mathrm{mM}$ phosphate buffer ( $\mathrm{pH} 8$ ) with $10 \mu \mathrm{M}$ Thioflavin $\mathrm{T} \pm 0,0.02,0.2$ or $1.0 \mu \mathrm{M}$ wild-type or $\mathrm{R} 47 \mathrm{H}$ sTREM2 in a fluorescence plate reader with a 440-nm excitation filter and a 480-nm emission filter without stirring as previously described ${ }^{37}$.

\section{Membrane permeabilization assay}

Lipid vesicles were prepared as previously described ${ }^{38}$, using a 100:1 mixture of phospholipids 16:0-18:1 phosphatidylcholine and biotinylated lipids 18:1-12:0 Biotin phosphatidylcholine. The mean diameter of vesicles was $\sim 200 \mathrm{~nm}$ and were filled with $100 \mu \mathrm{M}$ Cal-520 dye using five freeze-and-thaw cycles. Non-incorporated Cal-520 dye was separated from the dye filled vesicles by size-exclusion chromatography. The vesicles were immobilized in biotin labelled PLL-g-PEG coated glass coverslips using a biotin-neutravidin linkage. Then coverslips were incubated with of $50 \mu \mathrm{L} \mathrm{Ca}{ }^{2+}$ containing buffer solution and different position of the coverslips imaged $\left(F_{\text {blank }}\right)$. Stage movements were controlled using an automated bean BeanShell-based program which allows fields-of-view selection without user bias. Then the $10 \mu \mathrm{M}$ aggregation (6hour time point) solution was added to the coverslips so that the final concentration of the peptide under the coverslip was $200 \mathrm{nM}$. Then the sample was incubated with the vesicles for 20 minutes and the same fields of view of each coverslip were imaged $\left(F_{\text {sample }}\right)$. Then, $10 \mu \mathrm{L}$ of 1 $\mathrm{mg} / \mathrm{mL}$ of ionomycin was added to the same coverslips and the same fields of view were imaged again ( $F_{\text {ionomycin }}$ ). lonomycin is a calcium ionophore and causes saturation of the fluorescence intensity observed. This allows us to normalise fluorescence originating from individual vesicles and subsequently quantify the sample induced $\mathrm{Ca}^{2+}$ influx using the following equation: $\quad$ Average $\mathrm{Ca}^{2+}$ influx $=\left(F_{\text {sample }}-F_{\text {blank }}\right) /\left(F_{\text {ionomycin }}-F_{\text {blank }}\right)$

All the imaging was done using a Total Internal Reflection Fluorescence Microscope (TIRFM) based on an inverted Nikon Ti microscope. For excitation, a $488 \mathrm{~nm}$ laser beam was focused in the back focal plane of the 60x, 1.49 NA oil-immersion objective lens. Fluorescence signal emerged from the samples collected from the same objective and imaged air cooled EMCCD camera. All the imaging experiments were performed at 293K. Fluorescence images were acquired using a $488 \mathrm{~nm}$ laser $\left(\sim 10 \mathrm{~W} / \mathrm{cm}^{2}\right)$ for 50 frames with a scan speed of $20 \mathrm{~Hz}$ and analyzed using ImageJ to calculate the localized fluorescence intensity of each vesicle.

\section{Statistics}

All statistical analyses were performed using Prism (GraphPad Software Inc.). Nonparametric statistical tests were used when data did not show a normal distribution. The specific 
statistical test used in each experiment are described in the associated figure legend. Data are expressed as means \pm SEM. Results were considered significant if $p<0.05$. In most cases, the sample size (i.e. the number of biological repeats, $n$ ) was 3 , as indicated in the figure legends, and this number was selected as the default for experiments using isolated proteins, based on previous experience of variance sizes. In the case of Fig 3 ix, measuring the area of fibrils, the variance swas found to be high (because the number of fibrils is low and size large), so $\mathrm{n}$ was increased to 8 for $A \beta$ alone, but remained at $n=3$ for $A \beta+W T$ or R47H sTREM2. For Fig 4 iv, which is a primary cell culture experiment, the sample size was four, based on previous experience of variance sizes. The sample size $\mathrm{n}$ refers to biological repeats, which means repeating using a different biological sample preparation. Outliers were only encountered in the data of Fig 2 ii, and were identified and removed by using GraphPad Prism outlier calculator.

\section{Resource Availability}

The published article includes all data generated or analyzed during this study. Original source data for Figures in the paper are available upon request to the corresponding authors. No proprietary software was used in the data analysis. Further information and requests for resources and reagents should be directed to: gcb3@cam.ac.uk or phs22@cam.ac.uk

\section{Acknowledgements}

GCB, JW \& PStGH have received funding from the Innovative Medicines Initiative 2 Joint Undertaking under grant agreement No 115976 (PHAGO consortium), and from the Canadian Institute of Health Research (PStGH \& PEF), Canadian Consortium on Neurodegeneration in Aging, Wellcome Trust, Medical Research Council, Zenith Award Alzheimer Association (PStGH).

\section{Author Contributions}

AV, YZ, JS, JKG, KS, DHA, SD, MP, AB, MAB, RBD, FC, YZ, SQ, JH, JW and PEF performed and analysed the molecular biological, biochemical and cell biological experiments. KS and PEF performed and analyzed assembly/disassembly assay. KS and PEF performed and analyzed electron microscopy experiments, SEC and dot-blot assay. PF and DK performed and analysed the permeabilization assay. LMN, AV and SFL performed and analysed the single molecule imaging experiments. KS, ME, and PEF performed and analysed the biophysical binding assay. 
PSGH and GCB conceived and directed the experiments and acquired funding. All authors contributed to the writing of the manuscript.

\section{Declaration of Interests}

The authors have no conflicts of interests to declare.

\section{References}

1. Wong CW, Quaranta V, Glenner GG. Neuritic plaques and cerebrovascular amyloid in Alzheimer disease are antigenically related. Proc Natl Acad Sci U S A. 1985;82:8729-32. 2. Edwards FA. A Unifying Hypothesis for Alzheimer's Disease: From Plaques to Neurodegeneration. Trends Neurosci. 2019;42:310-322. doi: 10.1016/j.tins.2019.03.003 3. Yuan P, Condello C, Keene CD, Wang Y, Bird TD, Paul SM, Luo W, Colonna M, Baddeley D, Grutzendler J. TREM2 Haplodeficiency in Mice and Humans Impairs the Microglia Barrier Function Leading to Decreased Amyloid Compaction and Severe Axonal Dystrophy. Neuron. 2016;90:724-39. doi: 10.1016/j.neuron.2016.05.003.

4. Guerreiro R et al. TREM2 variants in Alzheimer's disease. N Engl J Med 2013;368, 117-127, doi:10.1056/NEJMoa1211851.

5. Jonsson T et al. Variant of TREM2 associated with the risk of Alzheimer's disease. N Engl J Med. 2013;368, 107-116, doi:10.1056/NEJMoa1211103.

6. Sims $R$ et al. Rare coding variants in PLCG2, ABI3, and TREM2 implicate microglialmediated innate immunity in Alzheimer's disease. Nat Genet 2017;49, 1373-1384, doi:10.1038/ng.3916.

7. Wunderlich P, Glebov K, Kemmerling N, Tien NT, Neumann H, Walter J. Sequential proteolytic processing of the triggering receptor expressed on myeloid cells-2 (TREM2) protein by ectodomain shedding and $\mathrm{y}$-secretase-dependent intramembranous cleavage. J Biol Chem. 2013;288:33027-36. doi: 10.1074/jbc.M113.517540.

8. Thornton P, Sevalle J, Deery MJ, Fraser G, Zhou Y, Ståhl S, Franssen EH, Dodd RB, Qamar S, Gomez Perez-Nievas B, Nicol LS, Eketjäll S, Revell J, Jones C, Billinton A, St George-Hyslop $\mathrm{PH}$, Chessell I, Crowther DC. TREM2 shedding by cleavage at the H157-S158 bond is accelerated for the Alzheimer's disease-associated H157Y variant. EMBO Mol Med. 2017;9:1366-1378. doi: 10.15252/emmm.201707673.

9. Schlepckow K, Kleinberger G, Fukumori A, Feederle R, Lichtenthaler SF, Steiner H, Haass C. An Alzheimer-associated TREM2 variant occurs at the ADAM cleavage site and affects 
shedding and phagocytic function. EMBO Mol Med. 2017;9:1356-1365. doi:

10.15252/emmm.201707672.

10. Suárez-Calvet M et al. Early increase of CSF sTREM2 in Alzheimer's disease is associated with tau related-neurodegeneration but not with amyloid- $\beta$ pathology. Mol Neurodegener. 2019;14:1. doi: 10.1186/s13024-018-0301-5.

11. Suarez-Calvet $M$ et al. Early changes in CSF sTREM2 in dominantly inherited Alzheimer's disease occur after amyloid deposition and neuronal injury. Sci Transl Med 2016;8:369ra178, doi:10.1126/scitranslmed.aag1767.

12. Suarez-Calvet $M$ et al. sTREM2 cerebrospinal fluid levels are a potential biomarker for microglia activity in early-stage Alzheimer's disease and associate with neuronal injury markers. EMBO Mol Med 2016;8:466-476. doi:10.15252/emmm.201506123.

13. Zhao $Y$ et al. TREM2 Is a Receptor for beta-Amyloid that Mediates Microglial Function. Neuron 2018;97:1023-1031. doi:10.1016/j.neuron.2018.01.031.

14. Zhong L, Wang Z, Wang D, Wang Z, Martens YA, Wu L, Xu Y, Wang K, Li J, Huang R, Can $\mathrm{D}, \mathrm{Xu} \mathrm{H}, \mathrm{Bu} \mathrm{G}$, Chen XF. Amyloid-beta modulates microglial responses by binding to the triggering receptor expressed on myeloid cells 2 (TREM2). Mol Neurodegener. 2018;13:15. 15. Lessard CB, Malnik SL, Zhou Y, Ladd TB, Cruz PE, Ran Y, Mahan TE, Chakrabaty P, Holtzman DM, Ulrich JD, Colonna M, Golde TE. High-affinity interactions and signal transduction between A oligomers and TREM2. EMBO Mol Med. 2018;10:e9027.

16. Zhong L, Xu Y, Zhuo R, Wang T, Wang K, Huang R, Wang D, Gao Y, Zhu Y, Sheng X, Chen K, Wang N, Zhu L, Can D, Marten Y, Shinohara M, Liu CC, Du D, Sun H, Wen L, Xu H, Bu G, Chen XF. Soluble TREM2 ameliorates pathological phenotypes by modulating microglial functions in an Alzheimer's disease model. Nat Commun. 2019;10:1365.

17. Parhizkar S, Arzberger T, Brendel M, Kleinberger G, Deussing M, Focke C, Nuscher B, Xiong M, Ghasemigharagoz A, Katzmarski N, Krasemann S, Lichtenthaler SF, Müller SA, Colombo A, Monasor LS, Tahirovic S, Herms J, Willem M, Pettkus N, Butovsky O, Bartenstein P, Edbauer D, Rominger A, Ertürk A, Grathwohl SA, Neher JJ, Holtzman DM, Meyer-Luehmann $\mathrm{M}$, Haass $\mathrm{C}$. Loss of TREM2 function increases amyloid seeding but reduces plaque-associated ApoE. Nat Neurosci. 2019;22:191-204.

18. Wang Y, Ulland TK, Ulrich JD, Song W, Tzaferis JA, Hole JT, Yuan P, Mahan TE, Shi Y, Gilfillan S, Cella M, Grutzendler J, DeMattos RB, Cirrito JR, Holtzman DM, Colonna M. TREM2mediated early microglial response limits diffusion and toxicity of amyloid plaques. J Exp Med. 2016;213:667-75. doi: 10.1084/jem.20151948. 
19. Ma LZ, Tan L, Bi YL, Shen XN, Xu W, Ma YH, Li HQ, Dong Q, Yu JT. Dynamic changes of CSF sTREM2 in preclinical Alzheimer's disease: the CABLE study. Mol Neurodegener. 2020;15:25. doi: 10.1186/s13024-020-00374-8.

20. Ewers $\mathrm{M}$ et al. Increased soluble TREM2 in cerebrospinal fluid is associated with reduced cognitive and clinical decline in Alzheimer's disease. Sci Transl Med. 2019;11:507. doi:

10.1126/scitransImed.aav6221.

21. Franzmeier N, Suárez-Calvet M, Frontzkowski L, Moore A, Hohman TJ, Morenas-Rodriguez E, Nuscher B, Shaw L, Trojanowski JQ, Dichgans M, Kleinberger G, Haass C, Ewers M; Alzheimer's Disease Neuroimaging Initiative (ADNI). Higher CSF sTREM2 attenuates ApoE4related risk for cognitive decline and neurodegeneration. Mol Neurodegener. 2020;15:57. doi: 10.1186/s13024-020-00407-2.

22. Ewers M, Biechele G, Suárez-Calvet M, Sacher C, Blume T, Morenas-Rodriguez E, Deming Y, Piccio L, Cruchaga C, Kleinberger G, Shaw L, Trojanowski JQ, Herms J, Dichgans M; Alzheimer's Disease Neuroimaging Initiative (ADNI), Brendel M, Haass C, Franzmeier N. Higher CSF sTREM2 and microglia activation are associated with slower rates of beta-amyloid accumulation. EMBO Mol Med. 2020;12:e12308. doi: 10.15252/emmm.202012308.

23. Horrocks $\mathrm{MH}$ et al. Single-Molecule Imaging of Individual Amyloid Protein Aggregates in Human Biofluids. ACS Chem Neurosci 2016;7:399-406. doi:10.1021/acschemneuro.5b00324. 24. Concepcion $\mathrm{J}$ et al. Label-free detection of biomolecular interactions using Bio-Layer Interferometry for kinetic characterization. Comb Chem High Throughput Screen 2009;12:791800.

25. Harper JD, Wong SS, Lieber CM, Lansbury PT Jr. Assembly of A beta amyloid protofibrils: an in vitro model for a possible early event in Alzheimer's disease. Biochemistry. 1999 Jul 13;38(28):8972-80.

26. Nichols MR1, Moss MA, Reed DK, Cratic-McDaniel S, Hoh JH, Rosenberry TL. Amyloidbeta protofibrils differ from amyloid-beta aggregates induced in dilute hexafluoroisopropanol in stability and morphology. J Biol Chem. 2005;280:2471-80.

27. De S, Whiten DR, Ruggeri FS, Hughes C, Rodrigues M, Sideris DI, Taylor CG, Aprile FA, Muyldermans S, Knowles TPJ, Vendruscolo M, Bryant C, Blennow K, Skoog I, Kern S, Zetterberg $\mathrm{H}$, Klenerman $\mathrm{D}$. Soluble aggregates present in cerebrospinal fluid change in size and mechanism of toxicity during Alzheimer's disease progression. Acta Neuropathol Commun. 2019;7:120.

28. De S, Wirthensohn DC, Flagmeier P, Hughes C, Aprile FA, Ruggeri FS, Whiten DR, Emin D, Xia Z, Varela JA, Sormanni P, Kundel F, Knowles TPJ, Dobson CM, Bryant C, Vendruscolo M, 
Klenerman D. Different soluble aggregates of $A \beta 42$ can give rise to cellular toxicity through different mechanisms. Nat Commun. 2019;10:1541.

29. Neniskyte U, Neher JJ, Brown GC. Neuronal death induced by nanomolar amyloid $\beta$ is mediated by primary phagocytosis of neurons by microglia. J Biol Chem. 2011;286:39904-13. 30. Piccio L, Deming Y, Del-Águila JL, Ghezzi L, Holtzman DM, Fagan AM, Fenoglio C, Galimberti D, Borroni B, Cruchaga C. Cerebrospinal fluid soluble TREM2 is higher in Alzheimer disease and associated with mutation status. Acta Neuropathol. 2016;131:925-33.

31. Yang T, Xu H, Walsh DM, Selkoe DJ. Large Soluble Oligomers of Amyloid $\beta$-Protein from Alzheimer Brain Are Far Less Neuroactive Than the Smaller Oligomers to Which They Dissociate. J Neurosci 2017; 35:152-163.

32. Iljina M, Hong L, Horrocks MH, Ludtmann MH, Choi ML, Hughes CD, Ruggeri FS, Guilliams T, Buell AK, Lee JE, Gandhi S, Lee SF, Bryant CE, Vendruscolo M, Knowles TPJ, Dobson CM, De Genst E, Klenerman D. Nanobodies raised against monomeric a-synuclein inhibit fibril formation and destabilize toxic oligomeric species. BMC Biol. 2017;15:57.

33. Kang SS et al. Behavioral and transcriptomic analysis of Trem2-null mice: not all knockout mice are created equal. Hum Mol Genet 2018;27:211-223. doi:10.1093/hmg/ddx366.

33. Bal-Price A, Brown GC. Inflammatory neurodegeneration mediated by nitric oxide from activated glia-inhibiting neuronal respiration, causing glutamate release and excitotoxicity. Journal of Neuroscience 2001;21:6480-6491.

34. Stine WB Jr, Dahlgren KN, Krafft GA, LaDu MJ. In vitro characterization of conditions for amyloid-beta peptide oligomerization and fibrillogenesis. J Biol Chem 2003;278:11612-11622. doi:10.1074/jbc.M210207200.

35. Drews A et al. Individual aggregates of amyloid beta induce temporary calcium influx through the cell membrane of neuronal cells. Sci Rep 2016;6:31910. doi:10.1038/srep31910. 36. Sevalle $\mathrm{J}$ et al. Aminopeptidase $\mathrm{A}$ contributes to the $\mathrm{N}$-terminal truncation of amyloid betapeptide. J Neurochem 2009;109:248-256. doi:10.1111/j.1471-4159.2009.05950.x.

37. Chia $S$ et al. Monomeric and fibrillar $\alpha$-synuclein exert opposite effects on the catalytic cycle that promotes the proliferation of A 42 aggregates. Proc. Natl. Acad. Sci. 2017;114:8005-8010. 38. Flagmeier $\mathrm{P}$ et al. Ultrasensitive measurement of $\mathrm{Ca}^{2+}$ influx into lipid vesicles induced by protein aggregates. Angew. Chem. Int. Ed. 2017;56:7750-7754. 
Supplementary Table 1.

Dissociation constants, on and off rates for WT and R47H sTREM2 binding to A $\beta$ oligomers. Fitted parameters for Supplementary Figures 6iii and iv.

\begin{tabular}{|l|c|c|}
\hline & WT & R47H \\
\hline $\mathbf{K}_{\mathbf{D}} \mathbf{1}(\boldsymbol{\mu M})$ & $2.00 \pm 0.15$ & $11.70 \pm 5.97$ \\
\hline $\mathbf{K}_{\mathbf{D}} \mathbf{2}(\boldsymbol{\mu M})$ & $0.29 \pm 0.08$ & $1.22 \pm 0.30$ \\
\hline $\mathbf{K}_{\text {on }} \mathbf{1}(\mathbf{1} / \mathbf{M s})$ & $217300 \pm 20200$ & $59660 \pm 3142$ \\
\hline $\mathbf{k}_{\text {off }} \mathbf{1}(\mathbf{1 / s})$ & $0.4353 \pm 0.0162$ & $0.6986 \pm 0.0304$ \\
\hline $\mathbf{k}_{\text {on }} \mathbf{2}(\mathbf{1} / \mathbf{M s})$ & $26250 \pm 3054$ & $6316 \pm 342$ \\
\hline $\mathbf{K}_{\text {off }} \mathbf{2}(\mathbf{1} / \mathbf{s})$ & $0.007717 \pm 0.001845$ & $0.007712 \pm 0.001861$ \\
\hline $\mathbf{R}_{\max } \mathbf{1}$ & 0.6728 & 0.6637 \\
\hline $\mathbf{R}_{\max } \mathbf{2}$ & 0.2158 & 0.1753 \\
\hline Full $\mathbf{R}^{\wedge} \mathbf{2}$ & 0.9951 & 0.9936 \\
\hline
\end{tabular}


Supplementary Figure 1

i)
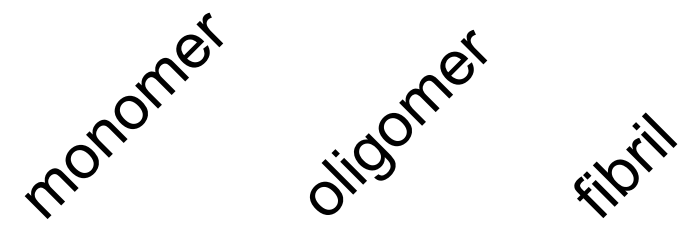

$6 e 10$

LOC

iii) ii)

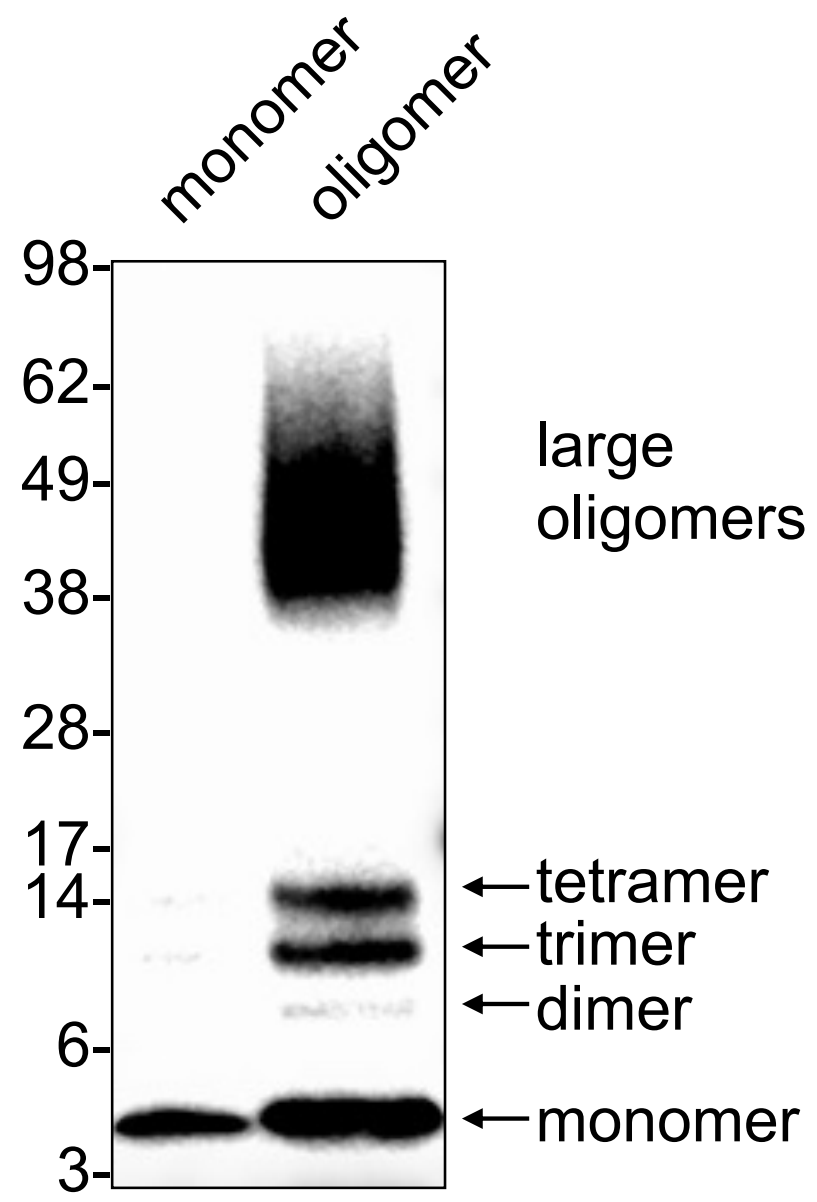




\section{Supplementary Figure 1. Characterization of $\boldsymbol{A} \boldsymbol{\beta}$ species,}

i \& ii): Characterization of $A \beta$ species. $A \beta 42$ monomers, oligomers and fibrils were prepared as previously described.

i) $20 \mathrm{ng}$ aliquots of $A \beta$ monomers, oligomers and fibrils were applied to a nitrocellulose membrane and probed with mouse anti-A $\beta$ antibody (6E10) or with rabbit anti-Amyloid fibrils antibody (LOC). ii) $A \beta$ monomers and oligomers were identified by Western blot analysis. $A \beta$ were separated by SDS-PAGE on a $4-12 \%$ NuPAGE bis-Tris gel and probed with anti-A $\beta$ antibody (6E10). iii \& iv) Characterization of $A \beta$ species used in $B L I$ experiments. Transmission electron microscopy (TEM) was performed using undiluted biotinylated $A \beta(100 \%)$ or a mixture of biotinylated $A \beta$ $(10 \%)$ and unlabelled $A \beta(90 \%)$ which were diluted to final concentrations of $1.1 \mu \mathrm{M}$. These TEM samples $(10 \mu \mathrm{l})$ were applied to carbonate coated grids and negatively stained with $1 \%$ phosphotungstic acid (PTA). TEM micrographs indicating the globular morphology of the $A \beta$ oligomers were obtained on a Hitachi $\mathrm{H}-7000$ operated at $75 \mathrm{kV}$. Scale bars are $100 \mathrm{~nm}$. 


\section{Supplementary Figure 2}

i)
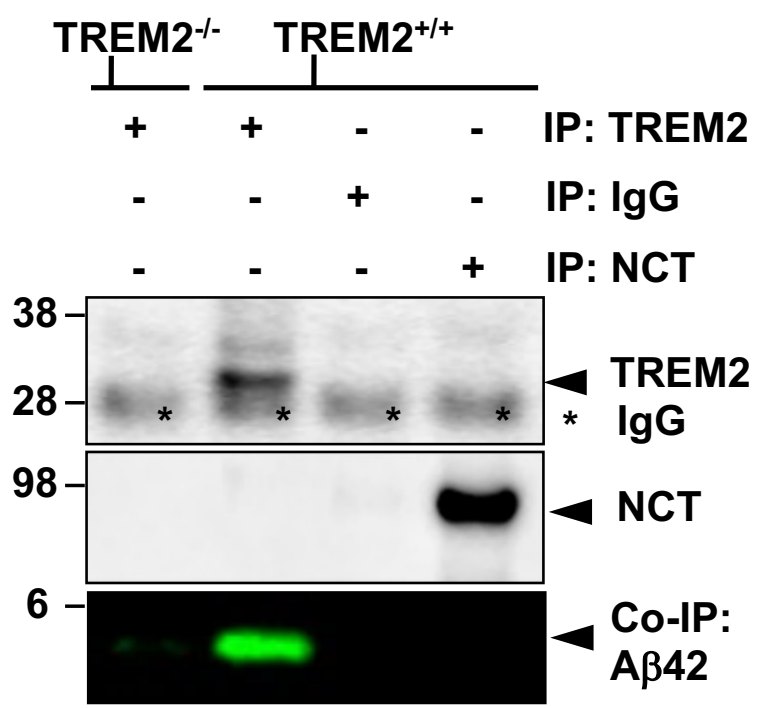

ii)

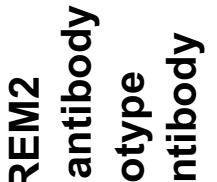

똔 응 음

+ +

ปับับ บั้

选 造施

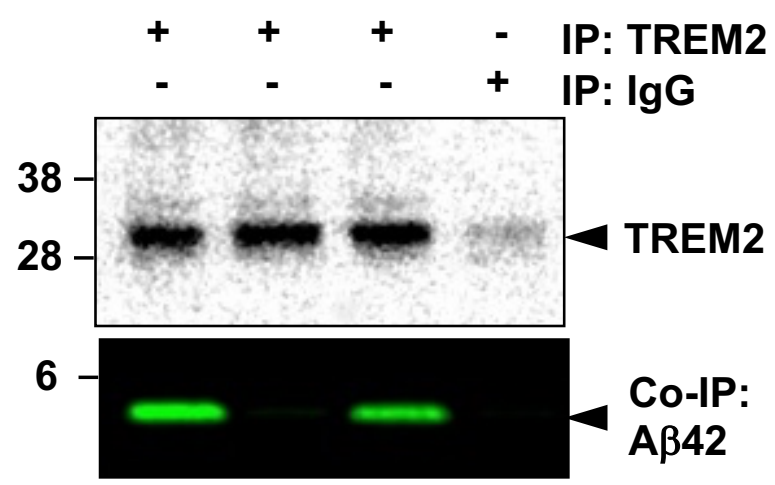

iii)

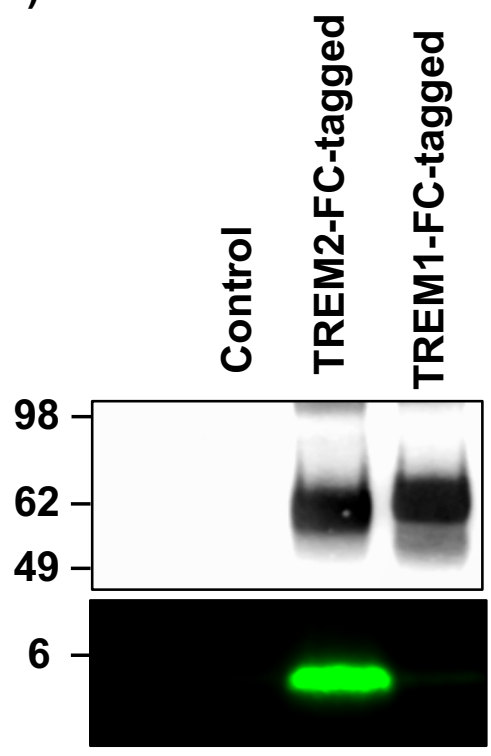

iv)
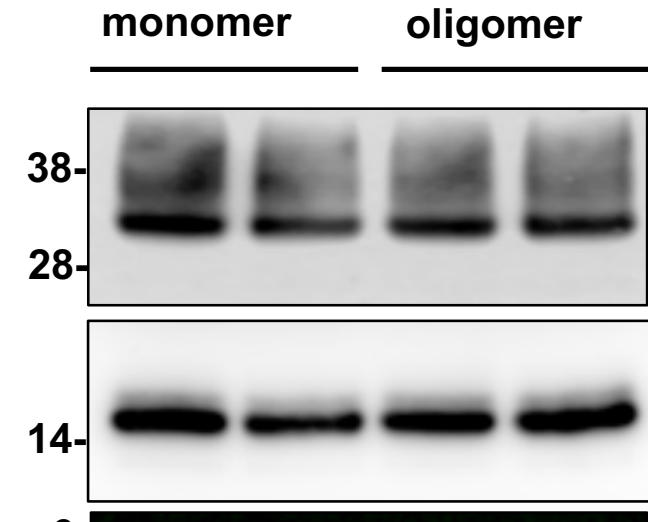

WB:

FC Tag

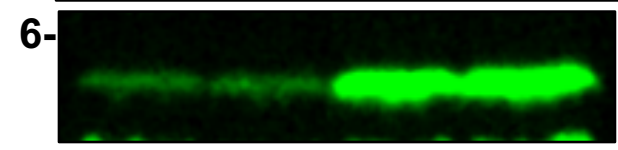

Co-IP:
A 342

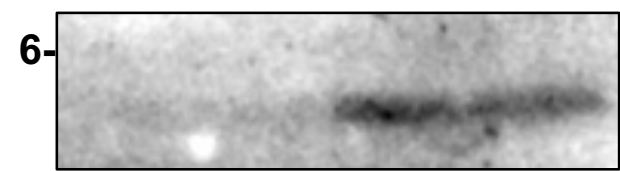

WB:

FLAG(DAP12)

Co-IP:

$A \beta 42$

WB:

FLAG(TREM2)

Co-IP WB:

$A \beta 42$

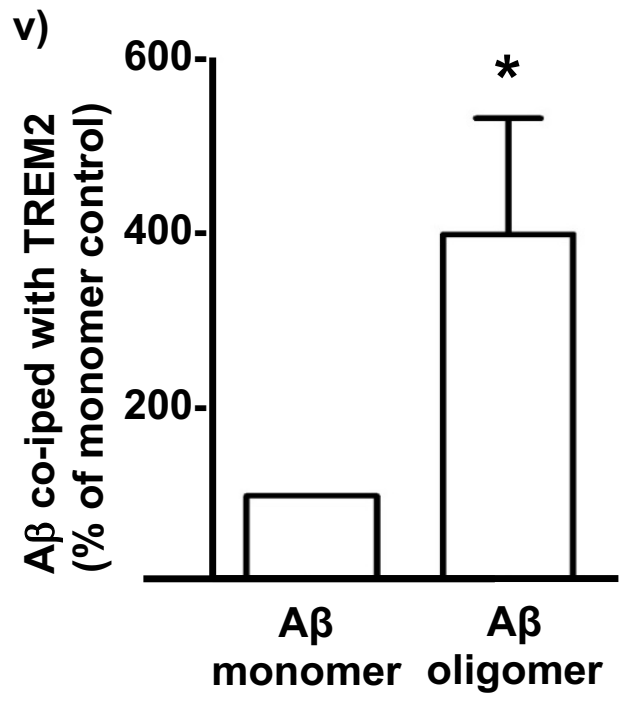




\section{Supplementary Figure 2. Full-length TREM2 binds $A \beta$ oligomers but not monomers.}

i) Primary microglia were incubated with $100 \mathrm{nM}$ HiLyte Fluor 647 -labelled $\mathrm{A} \beta 42$ at $4^{\circ} \mathrm{C}$ for 1 hour, then lysed, immunoprecipitated with anti-TREM 2 antibodies, and examined by SDS-PAGE and western blotting. $A \beta$ oligomers co-immunoprecipitate with endogenous TREM2 in primary microglia (lane 2 ), but not with other natively endogenous Type I glycoproteins, such as nicastrin (lane 4) or with isotype control antibody (lane 3). TREM 2 knockout microglia failed to co-immunoprecipitate $A \beta$ (lane 1). ii) This interaction was blocked by preincubation with monoclonal anti-TREM2 blocking antibody (lane 2), but not by isotype control antibodies (lane 3). iii): $A \beta$ coprecipitated with recombinant FC-TREM2 (lane 2) but not FC-TREM1 (lane 3). iv) A oligomers bind to TREM2 significantly better than monomers. HeLa cells transfected with DAP12-FLAG plus TREM2-FLAG were co-incubated with $100 \mathrm{nM}$ freshly prepared monomeric or oligomeric Hilyte Fluor 647 -labelled $A \beta$ at $4^{\circ} \mathrm{C}$ for 1 hour. Prior to incubation, the concentration of monomer/oligomer were adjusted and tested according to fluorescence dot exposure to ensure total $A \beta$ concentrations were the same in the working solutions of monomeric and oligomeric $A \beta$ preparations. Cell membrane lysates were then immunoprecipitated with anti-hTREM 2 antibody. The IP products were western blotted and probed with the indicated antibody. v) $A \beta$ oligomers bind to TREM2 significantly better than monomers. Quantification of iv) with $n=4$ replicates in 2 independent experiments; $p=0.03$ Mann-Whitney $U$ test). Error bars = SEM. 
Supplementary Figure 3

i)

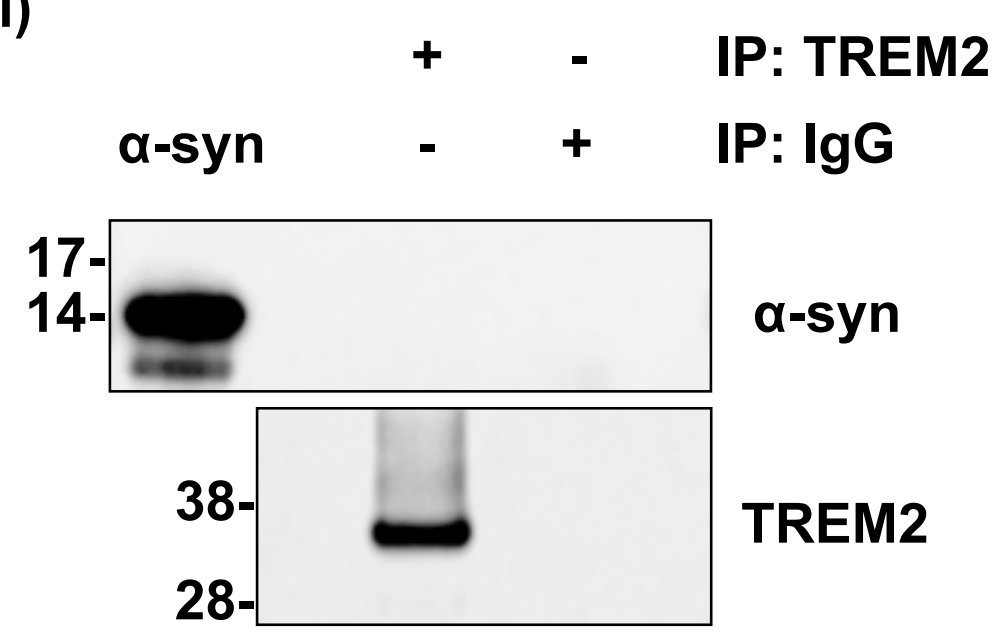

ii)

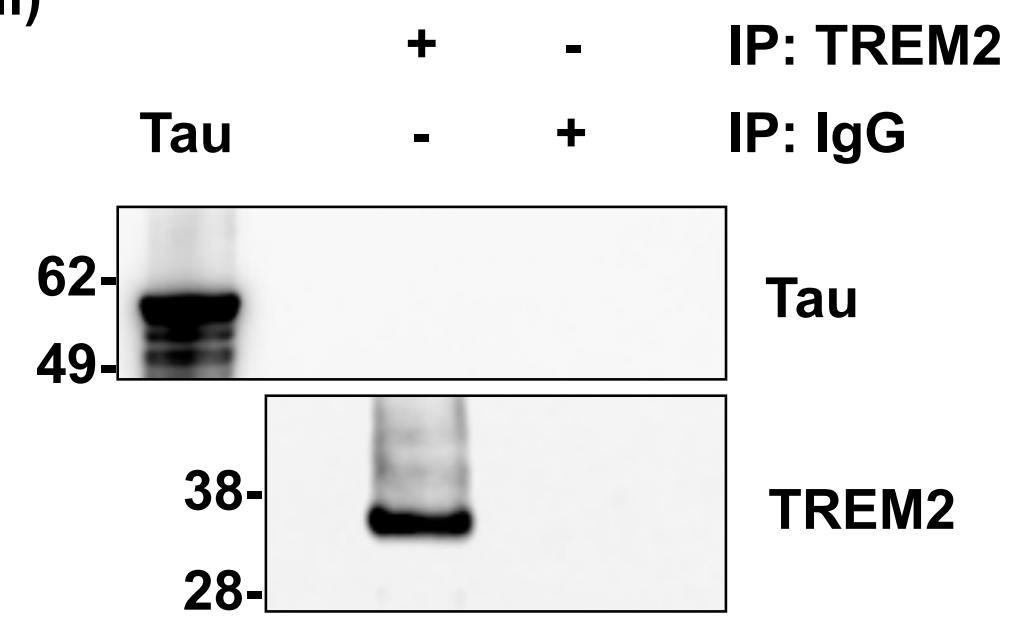

Supplementary Figure 3. TREM2 does not bind $\alpha$-synuclein or Tau oligomers. Primary microglia were incubated with $100 \mathrm{nM}$ oligomeric $\alpha$-synuclein or Tau protein at $4^{\circ} \mathrm{C}$ for 1 hour. Cell membrane lysates were then immunoprecipitated with human TREM 2 antibody or control IgG. The IP products were probed with the indicated antibody. 


\section{Supplementary Figure 4}

i)

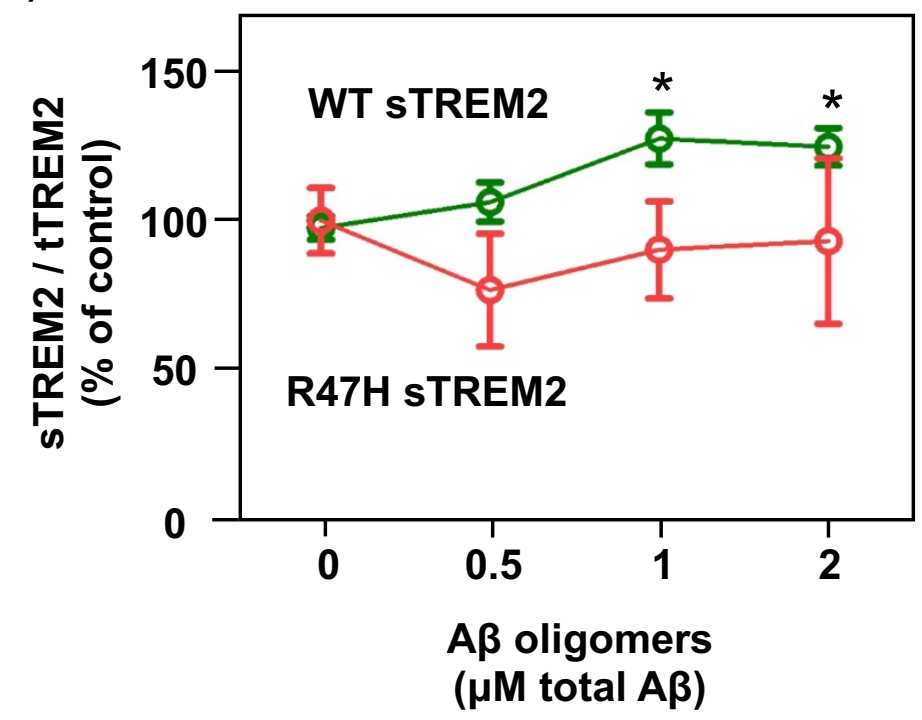

ii)

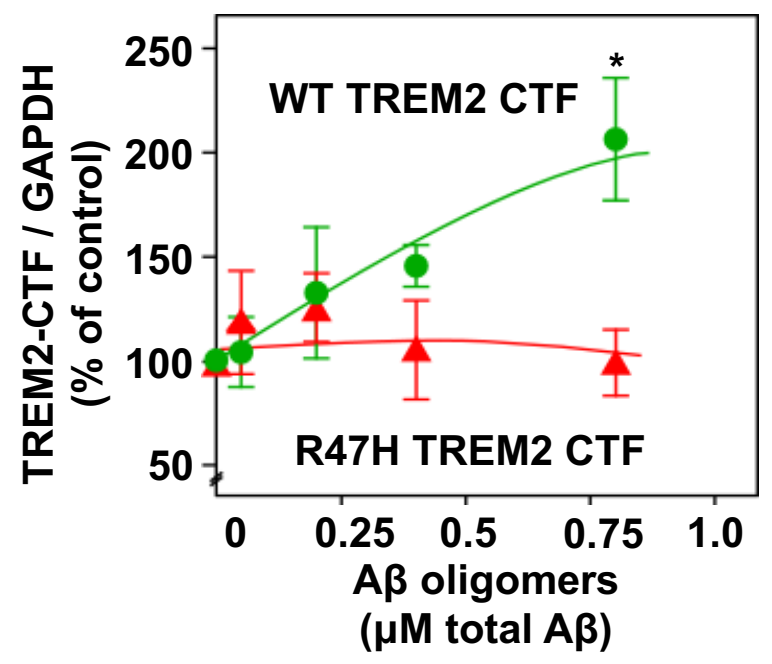

Supplementary Fig. 4. A $\beta$ oligomers induce sTREM2 release and TREM2-CTF accumulation in cells expressing wild-type (but not R47H) TREM2. i) In primary murine microglia, endogenously expressed wild type TREM2 (WT-TREM2, green line) showed dose-dependent increase in shedding of soluble sTREM2 extracellular domain (green line). A $\beta$ oligomers do not induce significant TREM2 endoproteolysis in microglia from homozygous CRISPR-Cas9 engineered R47H TREM2. Error bars $=\mathrm{SEM} ;{ }^{*}=\mathrm{p}<0.05, \mathrm{n}=4$ independent experiments with $\geq 10$ replications each; one-way ANOVA with Tukey's multiple comparisons post-test. ii) TREM2 membrane-bound C-terminal fragment was assayed (by western blots, Supplementary Fig. 5) 16 hours after addition of A $\beta$ oligomers to HEK293 cells overexpressing wild-type or R47H TREM2. 
Supplementary Figure 5

i) $\quad \mathrm{A} \beta$ monomers ( $\mathrm{nM}$ )

o 50200400800

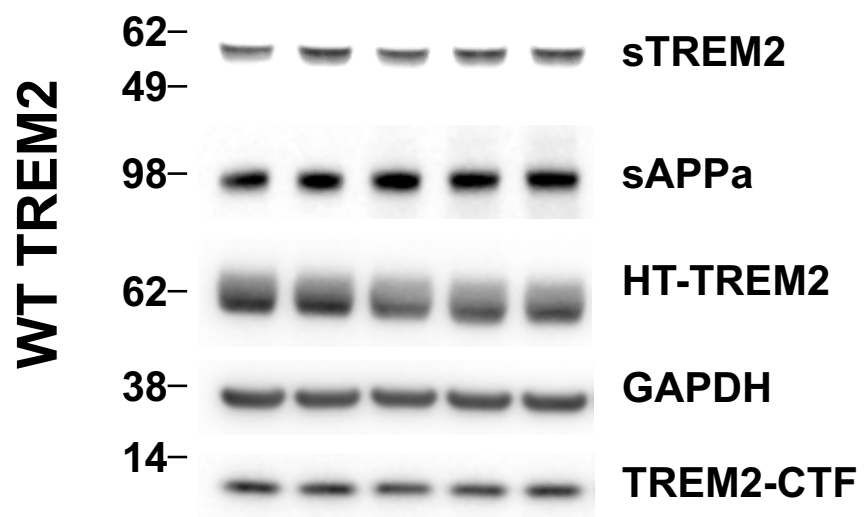

ii) $\quad \mathrm{A} \beta$ fibrils $(\mathrm{nM})$

$0 \quad 50200400800$

$62-$
$49-----$ STREM2
$98------$ SAPPa

62- $-E=-$ HT-TREM2

38- $-\infty-\infty$ GAPDH

14- iii) $A \beta$ oligomers ( $n M)$

0 50200400800

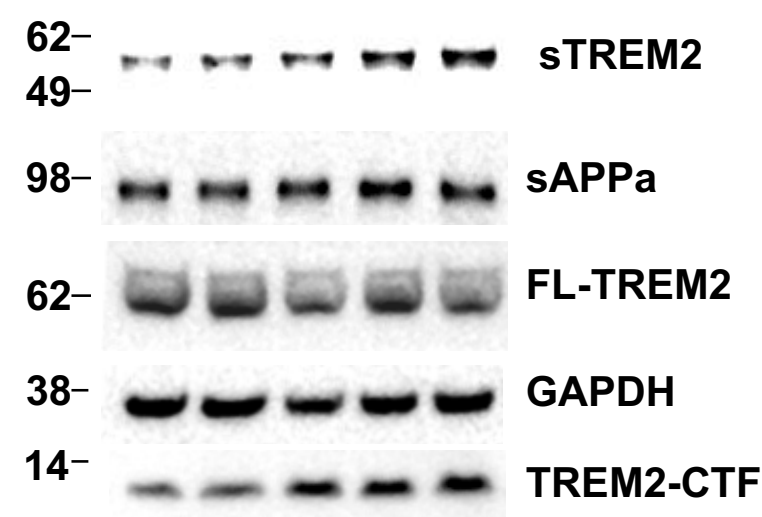

iv) $A \beta$ monomers ( $\mathrm{nM}$ )

$0 \quad 50200400800$

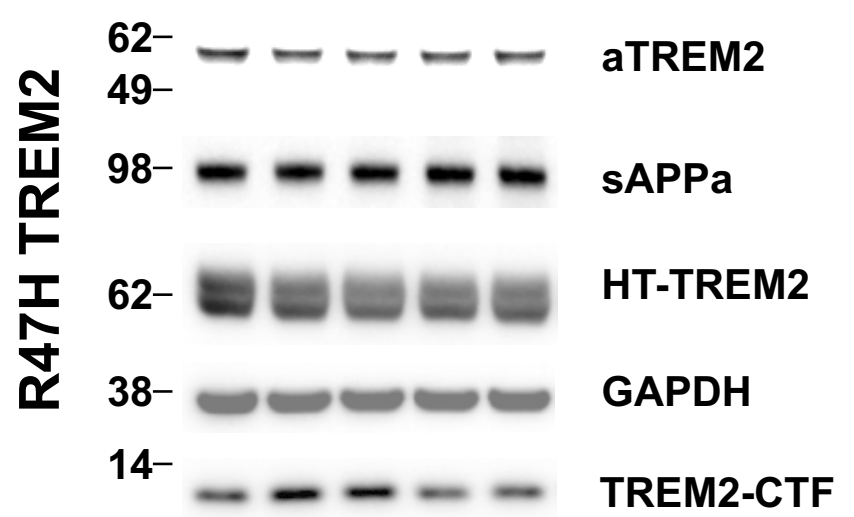

v) $\quad A \beta$ fibrils (nM)

o 50200400800

62- ---- STREM2

98- - - - SAPPa

62- 0 를

TREM2

38- - $-2-$ GAPDH

14-

TREM2-CTF

vi) $A \beta$ oligomers $(n M)$

o 50200400800

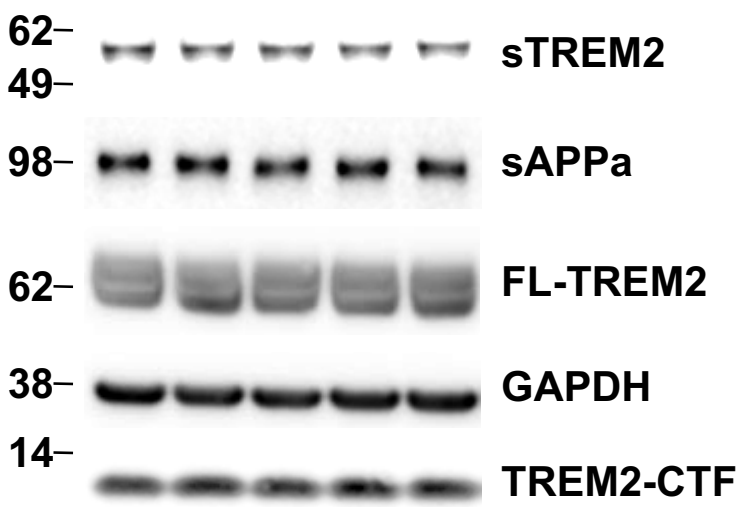

Supplementary Figure 5. A $\beta$ monomers and fibrils fail to trigger TREM2 cleavage, but $A \beta$ oligomers trigger TREM2 cleavage in wild-type but not R47H TREM2-expressing cells. $A \beta$ monomers ( $i$ \& iv) fibrils (ii \& v) and oligomers (iii \& vi) were added at the indicated concentrations to HEK293 cells co-expressing DAP12 and either wild-type (WT, I, ii \& iii) or R47H TREM2 (iv, v \& vi), both HaloTagged (HT-TREM2). TREM2 membrane-bound C-terminal fragment was assayed in cell lysates and sTREM 2 from the culture medium 16 hours after $A \beta$ addition. Representative western blots shown. 
Supplementary i) Figure 6

$A \beta$ monomer

$A \beta$ oligomer

Anti-TREM2

Anti-TREM2 (light exposure)

iii)

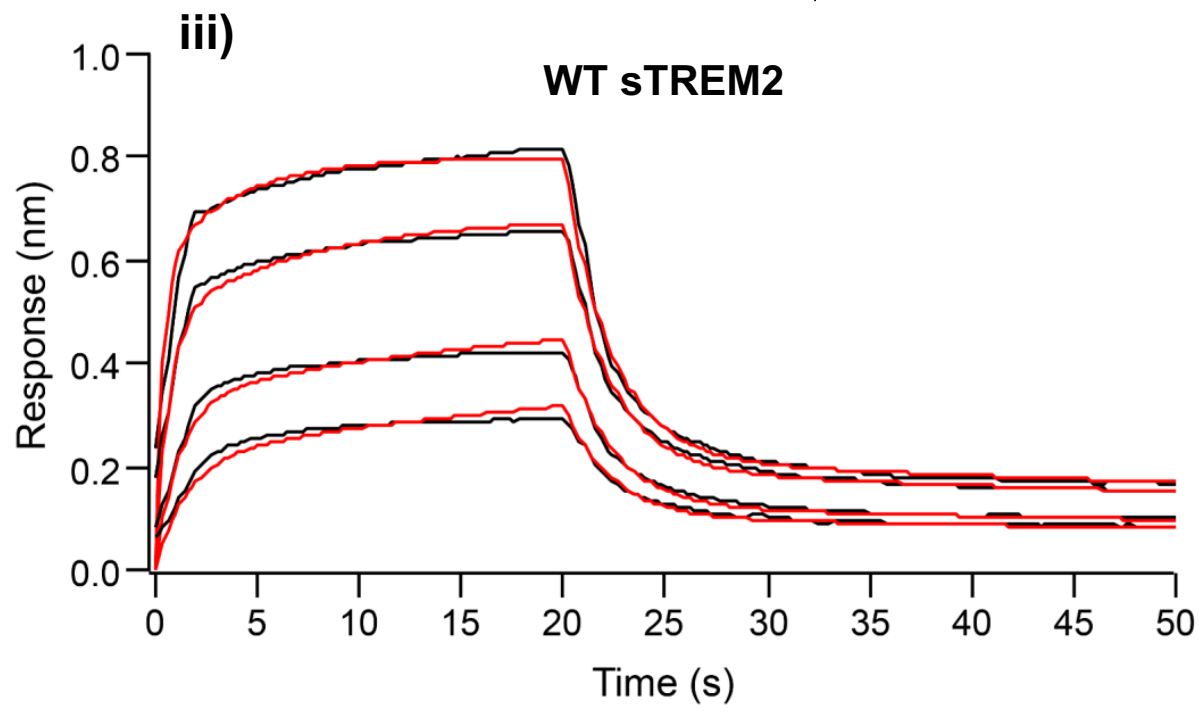

ii)
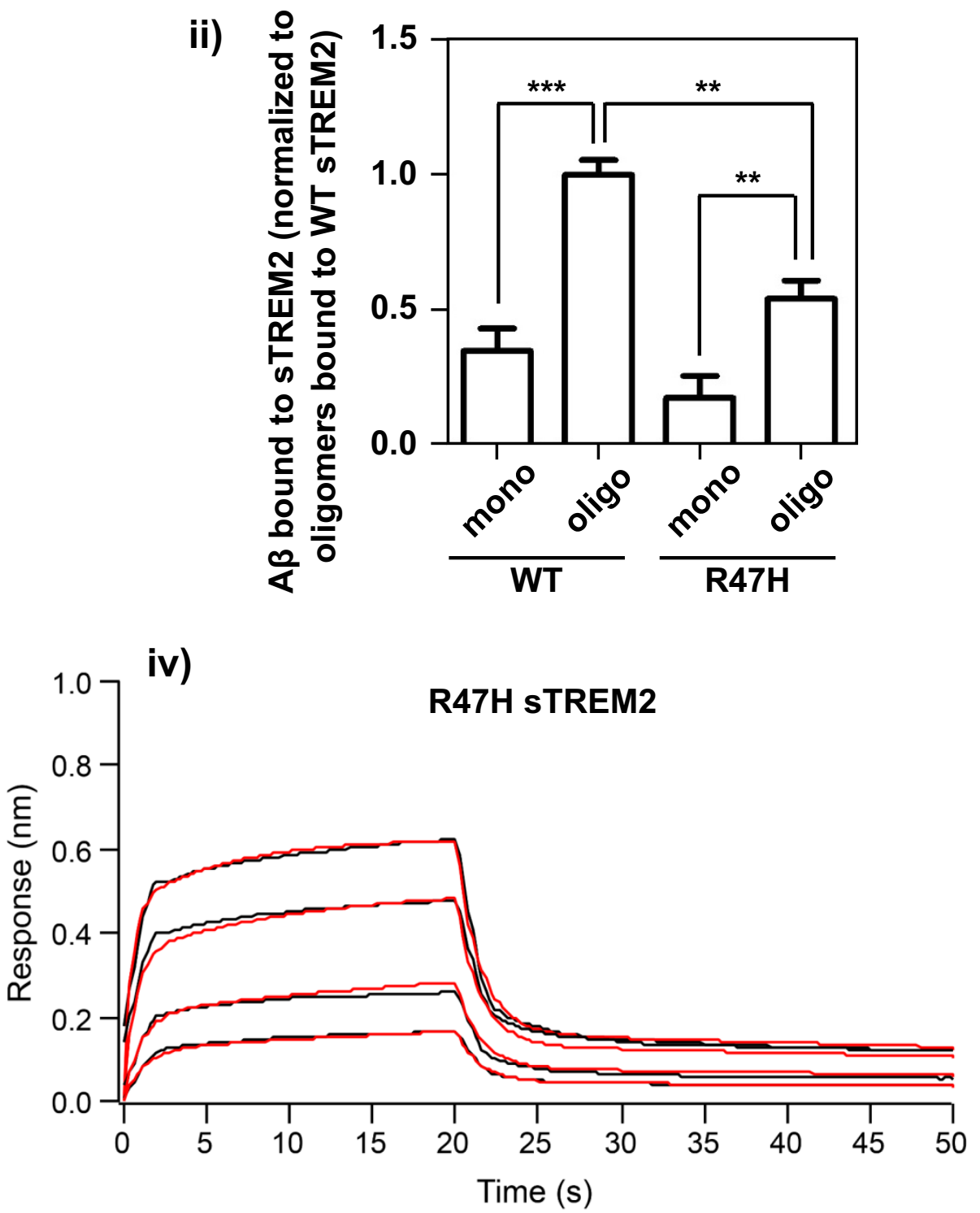

Supplementary Figure 6. Wild-type (WT) sTREM2 binds $A \beta$ oligomers more than $A \beta$ monomers, and R47H sTREM2 binds both less.

i) Dot Blot membrane strips spotted with $A \beta$ monomers, oligomers and anti-TREM2 antibody were probed with WT sTREM2-His (left) or R47H sTREM2-His (right). Lower left panel is a lighter exposure of the anti-TREM2 antibody showing that similar amounts of sTREM2 were used.

ii) Quantification of sTREM2 bound to A $\beta$. Error bars $=S E M ;{ }^{* *}=p<0.01,{ }^{* * *}=p<0.001, n=3$ independent experiments with 6 replications; oneway ANOVA with Tukey's post-hoc multiple comparisons test. iii) and iv) Bio-Layer Interferometry studies reveal a 2-state model for A $\beta$-binding to sTREM2. Curves of iii) WT sTREM2 or iv) R47H sTREM2 binding to A $\beta$ oligomers at four different concentrations.. Black lines indicate experimental data. Red lines indicate fitted curves from the 2:1 model. The fitted parameters are shown in Table 1. 


\section{Supplementary Figure 7}

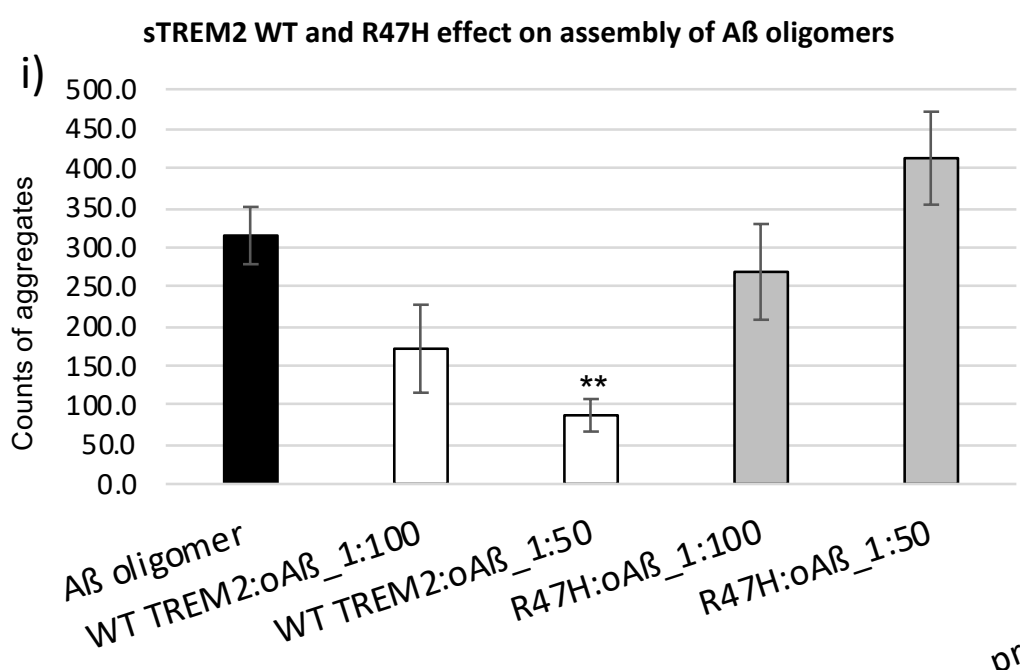

sTREM2 WT and R47H effect on disassembly of preformed Aß oligomers

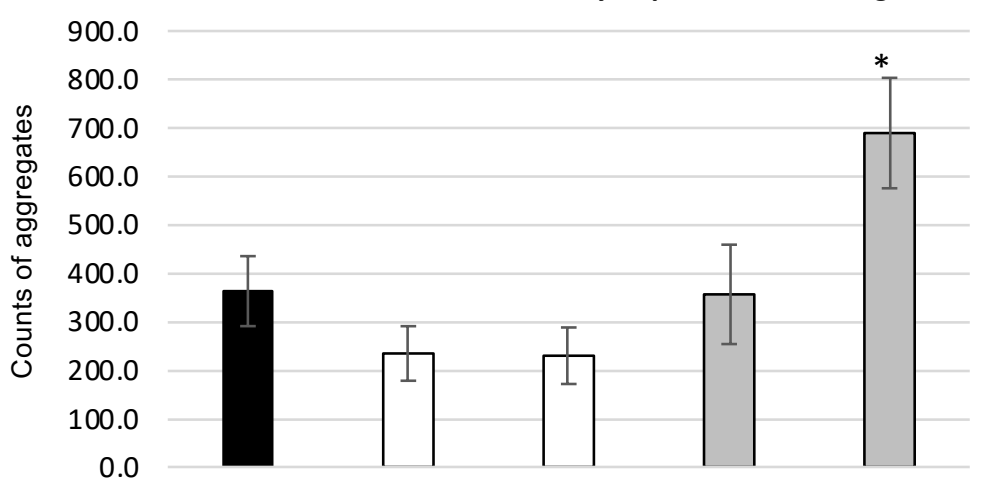

Supplementary Figure 7. WT sTREM2 decreases $A \boldsymbol{\beta}$ oligomer assembly, whereas R47H sTREM2 increases $A \beta$ oligomer disassembly. i) $A \beta$ was oligomerized in the presence of absence of WT or R47H sTREM2 (sTREM2 1:50 or 1:100 molar ratio to A $\beta$ ). After by transmission electron microscopy imaging (representative images in Fig 2 ), the numbers of $A \beta$ aggregates (oligomers) were counted. ii) $A \beta$ was oligomerized, and then incubated in the presence of absence of WT or R47H STREM2 (sTREM2 1:5 or 1:1 molar ratio to $A \beta$ ). After imaging (representative images in Fig 2), the numbers of $A \beta$ aggregates (oligomers) were counted. Error bars represent SD. Statistical analysis was performed using one-way ANOVA followed by Bonferroni's multiple comparison test $(n=3, * p<0.05$, $* * p<0.01$ vs $A \beta$ oligomer alone). 


\section{Supplementary Figure 8}

\section{i) STREM2 effects on A $\beta$ oligomerization}
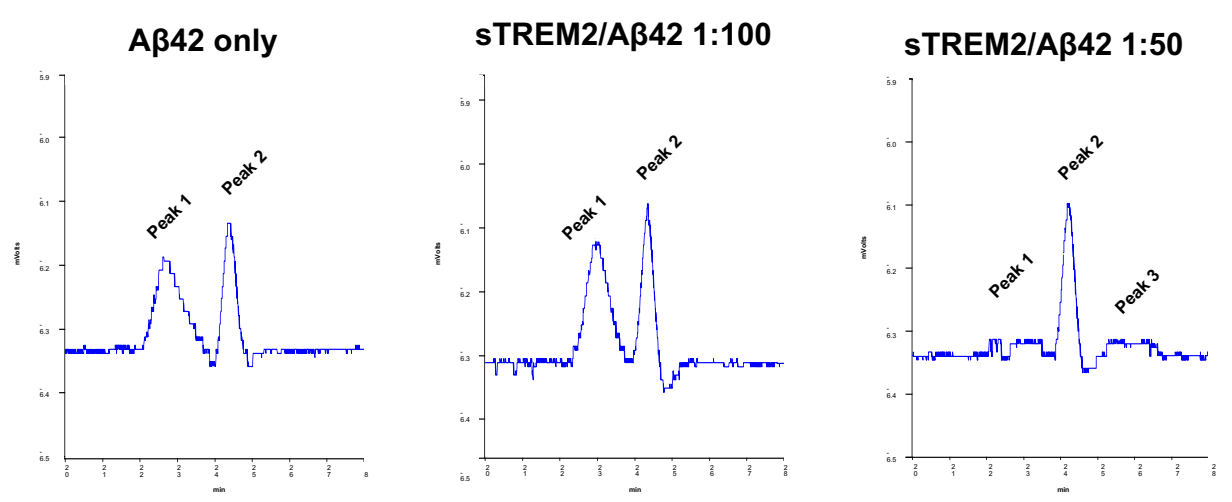

\begin{tabular}{|l|c|c|c|c|}
\hline Samples name & Peak & $\begin{array}{c}\text { peak area } \\
\text { (mVoltage*sec) }\end{array}$ & $\begin{array}{c}\text { peak width } \\
(\mathrm{sec})\end{array}$ & $\begin{array}{c}\text { retention time } \\
\text { (min) }\end{array}$ \\
\hline Aß42 only & Peak 1 & 8.01 & 44.4 & 22.61 \\
\hline STREM2/Aß42 1:100 & Peak 2 & 5.10 & 23.5 & 24.34 \\
\hline & Peak 1 & 7.80 & 41.1 & 22.94 \\
\hline STREM2/Aß42 1:50 & Peak 2 & 6.04 & 19.4 & 24.33 \\
\hline & Peak 1 & 1.15 & 51.2 & 22.81 \\
\hline & Peak 2 & 5.73 & 21.0 & 24.17 \\
\hline & Peak 3 & 3.07 & 70.2 & 25.30 \\
\hline
\end{tabular}

ii) sTREM2 effects on reversal of $A \beta$ oligomerization

A11

4G8
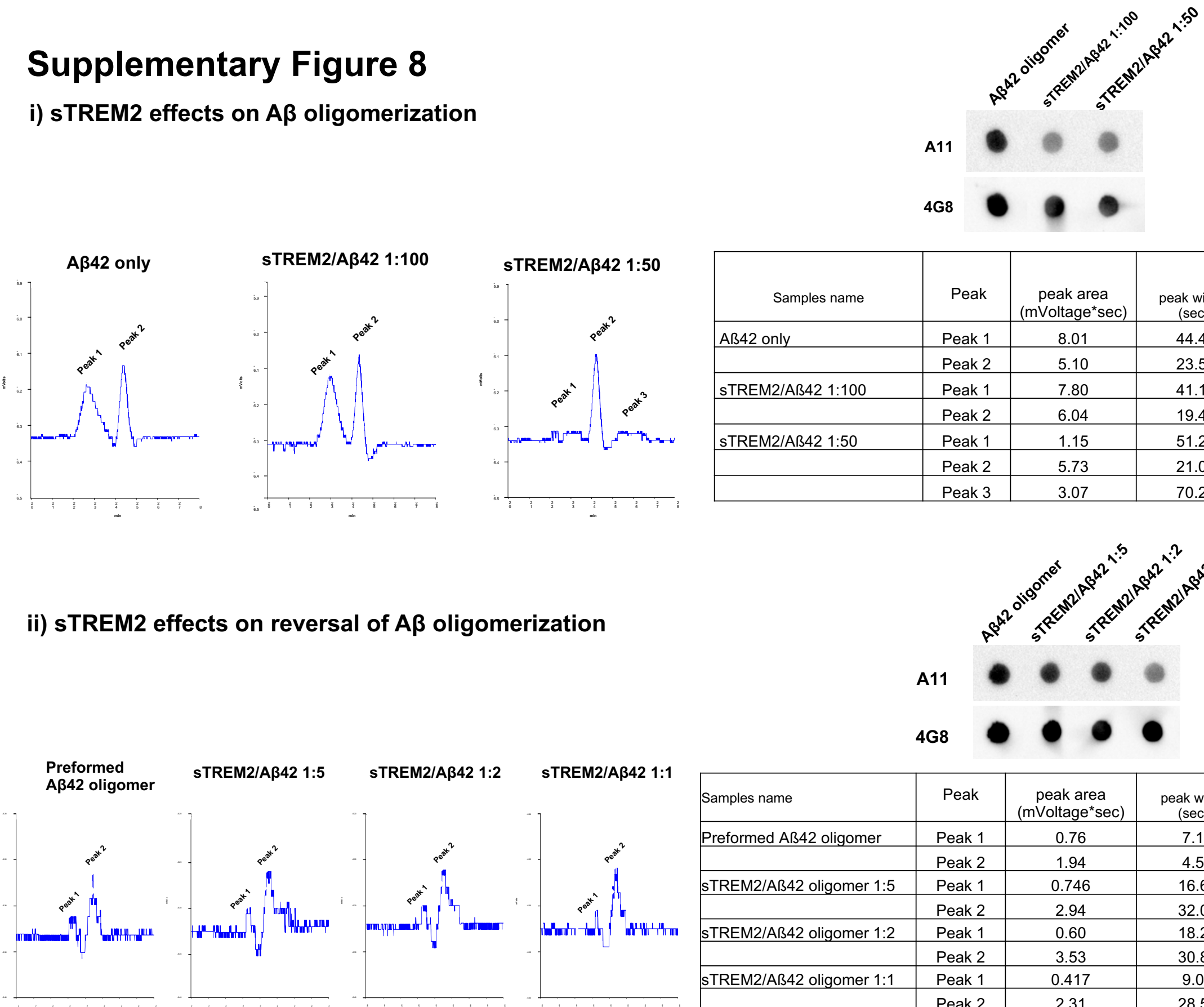

\begin{tabular}{|l|c|c|c|c|}
\hline \multicolumn{1}{|c|}{ A11 } \\
4G8
\end{tabular}


Supplementary Figure 8. Wild-type sTREM2 inhibits oligomerisation of monomeric $A \beta 42$, and disaggregates preformed $A \boldsymbol{\beta}$ oligomers. The same $A \beta$ preparations examined in Figure $2 \mathrm{i}$ by transmission electron microscopy were also investigated by HPLC-SEC (left panels and table). In both i) A $\beta$ oligomer formation and ii) A $\beta$ oligomer disaggregation experiments, sTREM2 reduced the area under "Peak 1", and increased the area under "Peak 2" and in some instances caused the appearance of a "Peak 3". The same $A \beta$ preparations were also examined by dot blot hybridisation against the A11 anti-oligomer antibody and the 4G8 anti-A $\beta$ antibody (right panels). In good agreement with both the TEM and the HPLC-SEC studies, the presence of STREM2 was associated with reductions in $A 11$ immunoreactive oligomeric $A \beta$ species. 


\section{Supplementary Figure 9}

i) STREM2 WT and R47H effect on assembly of Aß42 oligomers 600

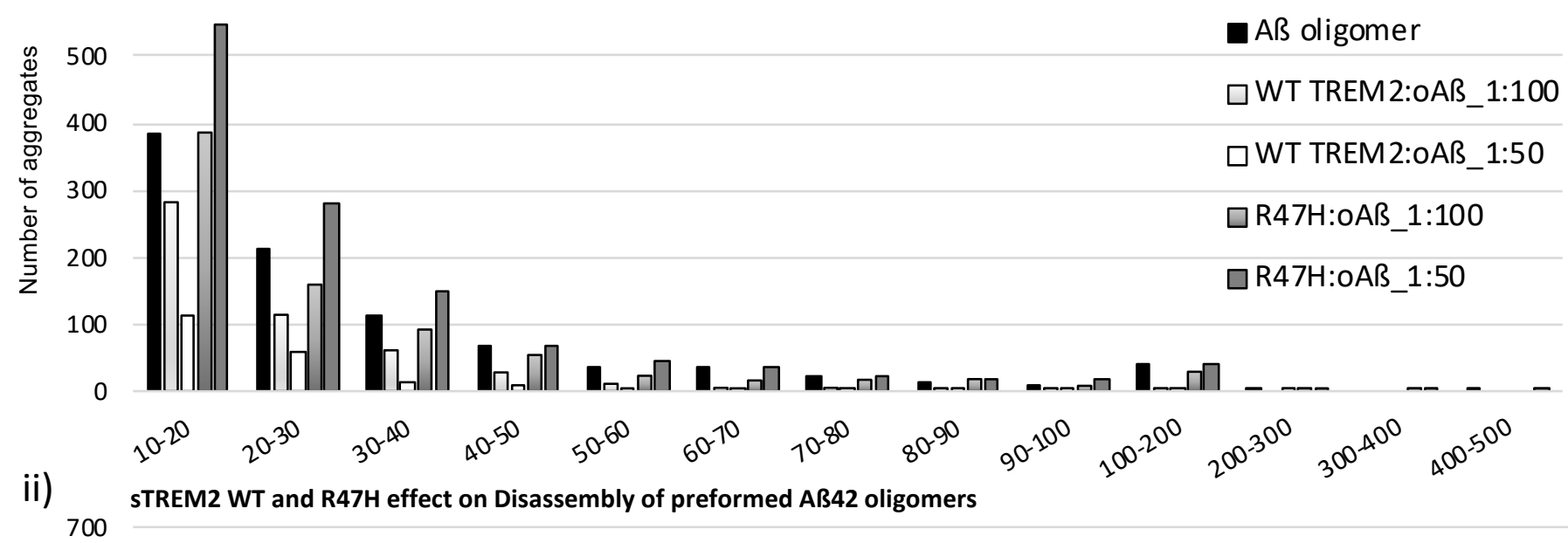

ii) 700 sTREM2 WT and R47H effect on Disassembly of preformed Aß42 oligomers

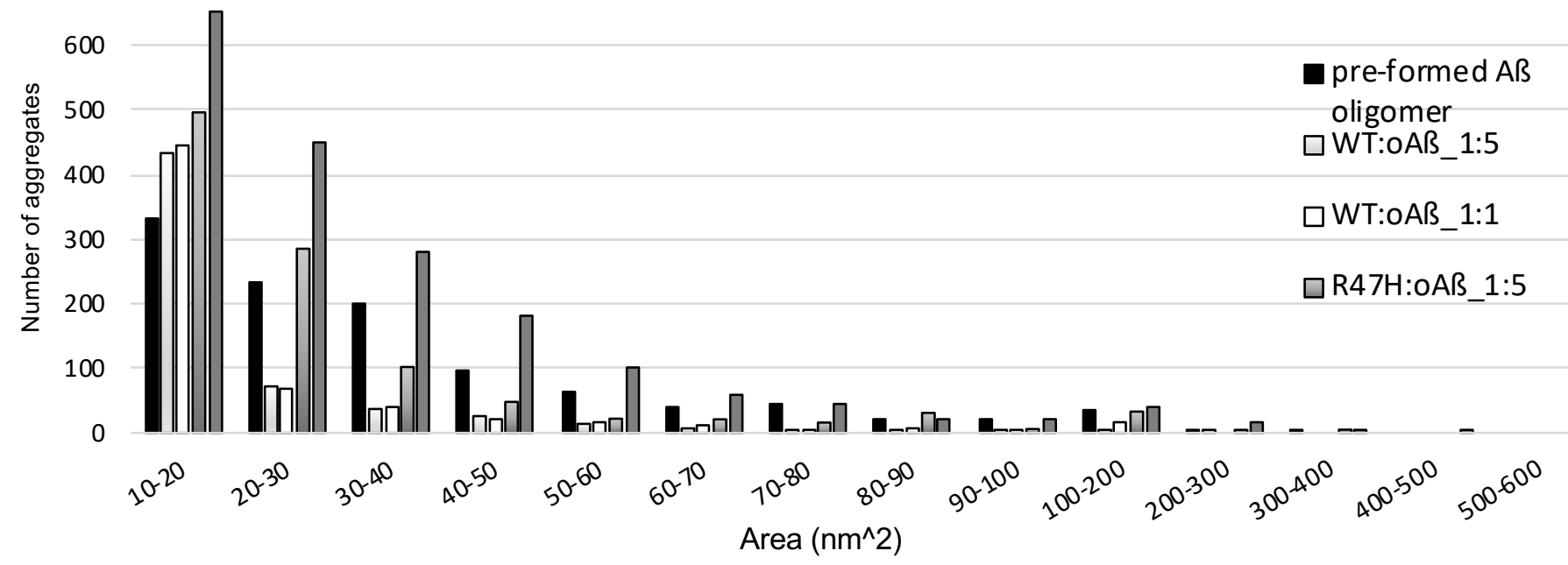

Supplementary Figure 9. WT sTREM2 preferentially inhibits the production of large $A \beta$ oligomers, and disaggregates large $\mathbf{A} \boldsymbol{\beta}$ oligomers into small oligomers; R47H sTREM2 increases the number of small $\mathbf{A} \boldsymbol{\beta}$ oligomers. i) Quantification of the size distribution of $A \beta$ aggregates (oligomers) produced by oligomerizing $A \beta$ in the presence or absence of WT or $\mathrm{R} 47 \mathrm{H}$ sTREM 2 at the indicated molar ratios. ii) Quantification of the size distribution of $A \beta$ aggregates (oligomers) resulting from incubation of preformed $A \beta$ oligomers \pm WT or R47H sTREM 2 at the indicated molar ratios. Representative TEM images in Fig 2. Data from three independent TEM images. 


\section{Supplementary Figure 10}

i)

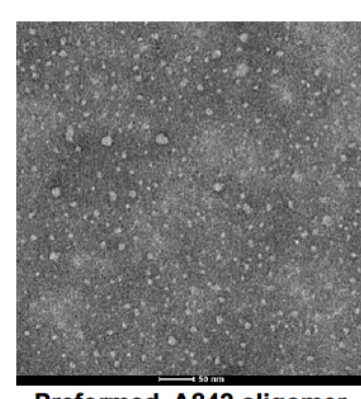

Preformed A $\beta 42$ oligomer (before incubation)

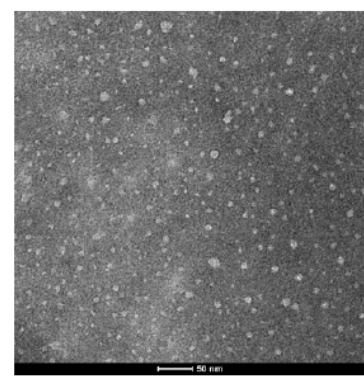

Preformed A $\mathbf{3 4 2}$ oligomer (after incubation)

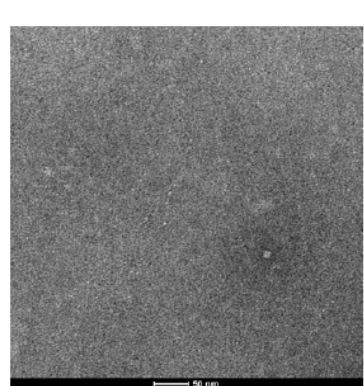

STREM2/Aß42 1:5

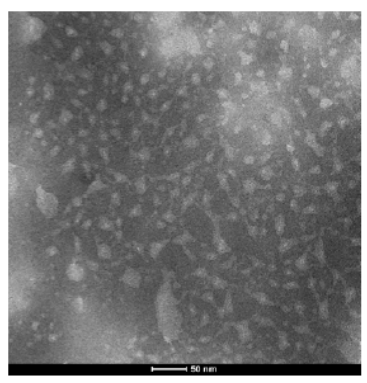

R47H/Aß42 1:5

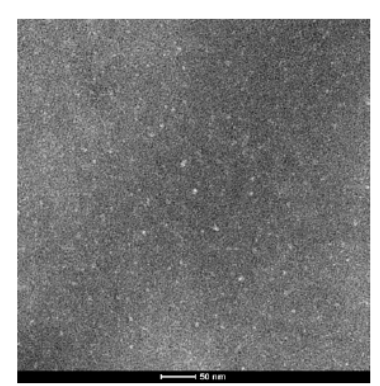

STREM2/Aß42 1:1

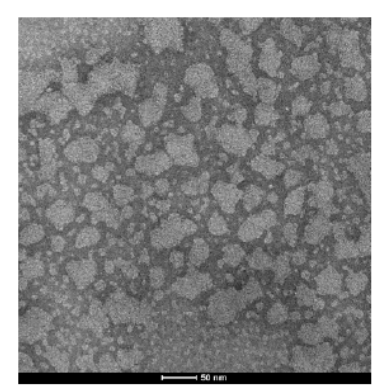

R47H/Aß42 1:1 ii)

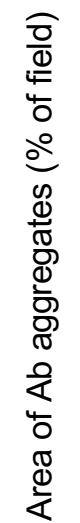

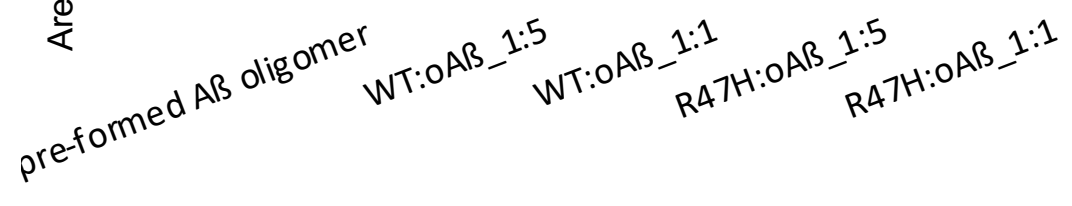

60.000

50.000

40.000

30.000

20.000

10.000

10.000
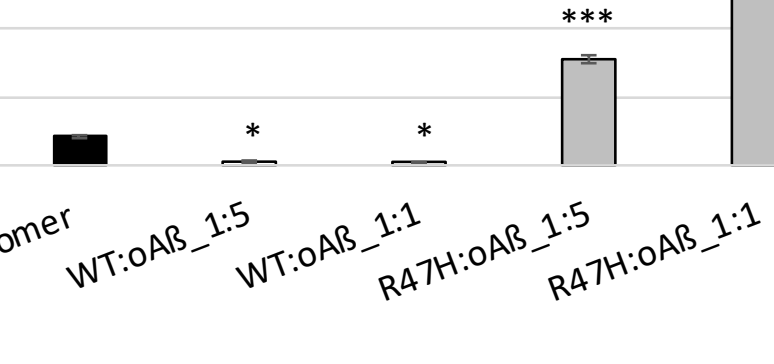

iii) 400

- pre-formed Aß

oligomer

$\square W T: O A ß \_1: 5$

$\square W T: O A ß \_1: 1$

$\square$ R47H:OAß_1:5
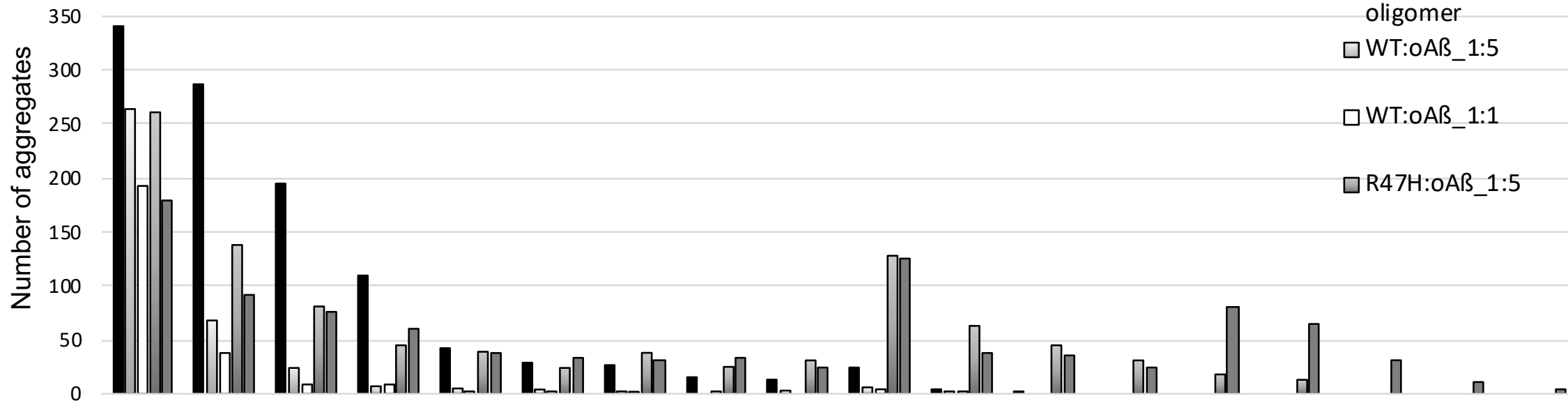

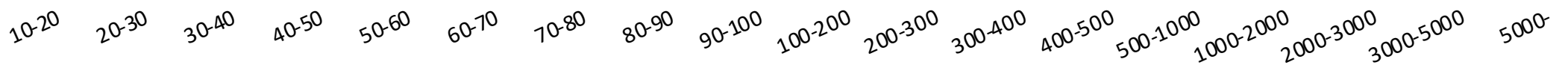

Size distribution of $A \beta$ aggregates. Area $\left(\mathrm{nm}^{\wedge} 2\right)$ 
Supplementary Figure 10. At low concentrations of $A \beta$, wild-type sTREM2 dissolves $A \beta$ oligomers, whereas $R 47 H$ sTREM2 induces very large $A \beta$ aggregates: total area and size distribution of $A \beta$ aggregates. Preformed $A \beta$ oligomers were incubated at $100 \mathrm{nM}$ $A \beta$ (monomer equivalent) \pm 20 or $100 \mathrm{nM}$ wild-type or $\mathrm{R} 47 \mathrm{H}$ sTREM2 for 30 mins at $37^{\circ} \mathrm{C}$, then TEM imaged. i) Representative TEM images. ii) Quantification of total area of $A \beta$ aggregates. Error bars represent SD. Statistical analysis was performed using one-way ANOVA followed by Bonferroni's multiple comparison test $\left(n=4,{ }^{*} p<0.05,{ }^{* * *} p<0.001 \mathrm{~A} \beta\right.$ oligomer alone). iii) Size distribution of the aggregates on from three independent TEM images. 


\section{Supplementary Figure 11}

i)

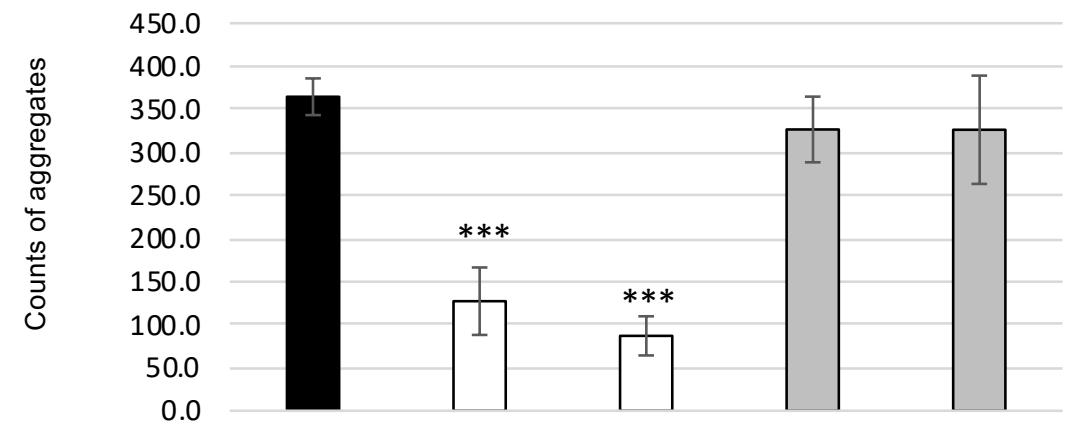

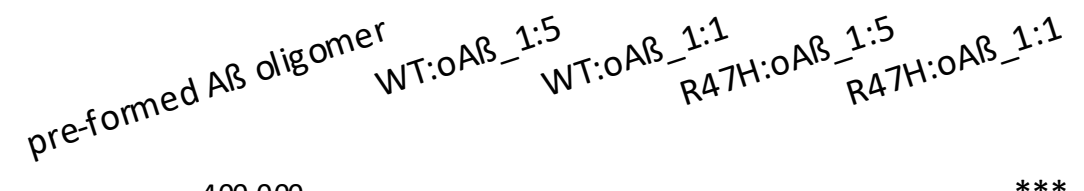

ii)

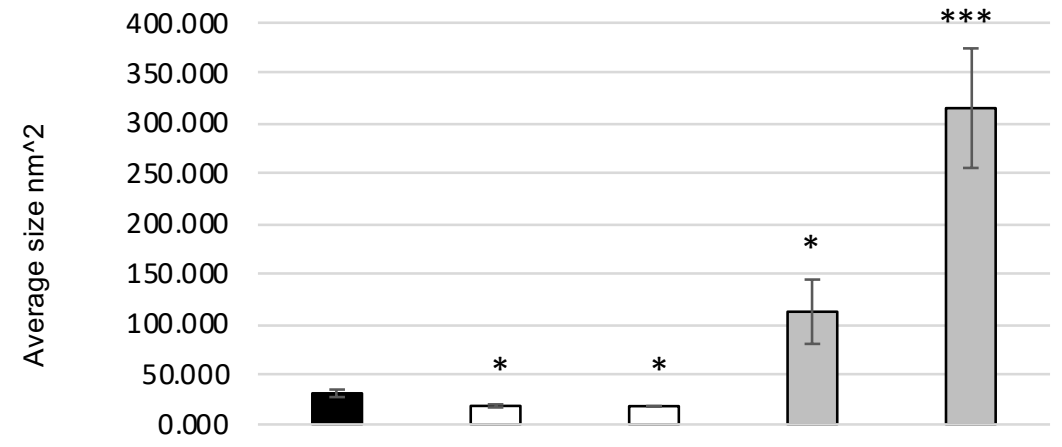

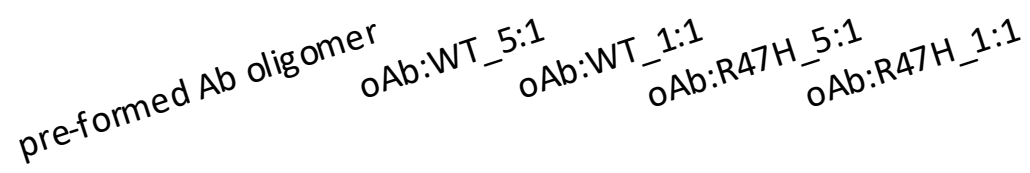

Supplementary Figure 11. At low concentrations of $A \beta$, wild-type sTREM2 dissolves $A \beta$ oligomers, whereas R47H sTREM2 induces very large $A \beta$ aggregates: numbers and sizes of $A \beta$ aggregates.

Further analysis of the aggregates of Supplementary Fig. 10. Preformed $A \beta$ oligomers were

incubated at $100 \mathrm{nM} \mathrm{A} \beta$ (monomer equivalent) \pm 20 or $100 \mathrm{nM}$ wild-type or R47H sTREM2 for 30 mins, then TEM imaged. i) Number of $A \beta$ aggregates. li) Average size (area) of each $A \beta$ aggregate. Error bars represent SD. Statistical analysis was performed using one-way ANOVA followed by Bonferroni's multiple comparison test ( $n=4,{ }^{*} p<0.05, * * * p<0.001$ A $\beta$ oligomer alone). 


\section{Supplementary Figure 12}

i)

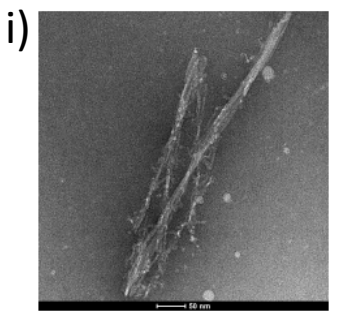

Preformed Aß42 fibril

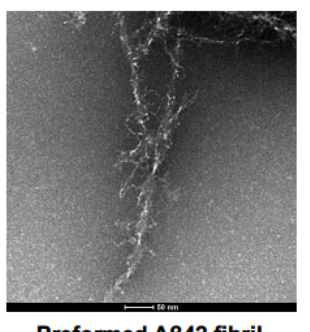

Preformed A $\beta 42$ fibril (after additional 24hr)

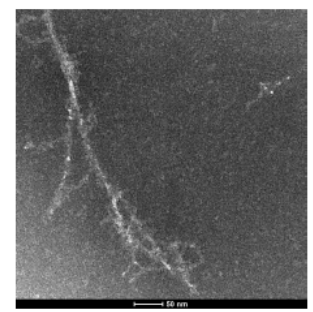

STREM2/Aß42 1:1 $30 \mathrm{~min}$

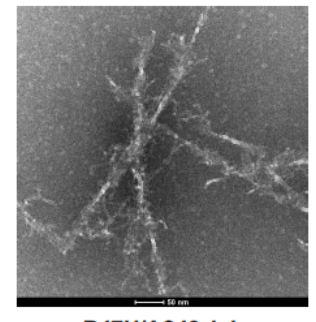

R47H/Aß42 1:1 $30 \mathrm{~min}$

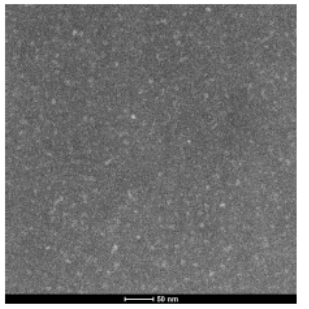

STREM2/Aß42 1:1 $2 \mathrm{hr}$

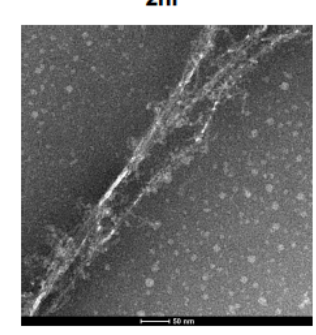

R47H/Aß42 1:1

$2 \mathrm{hr}$

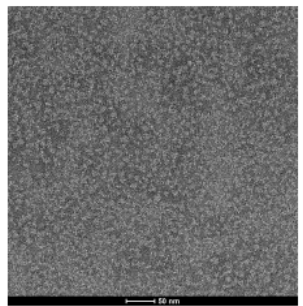

STREM2/Aß42 1:1 $24 \mathrm{~h}$

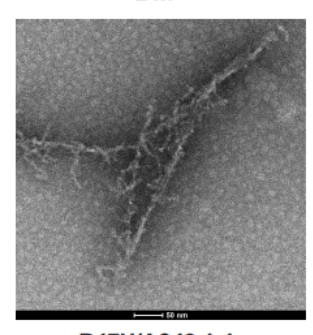

R47H/Aß42 1:1

24h
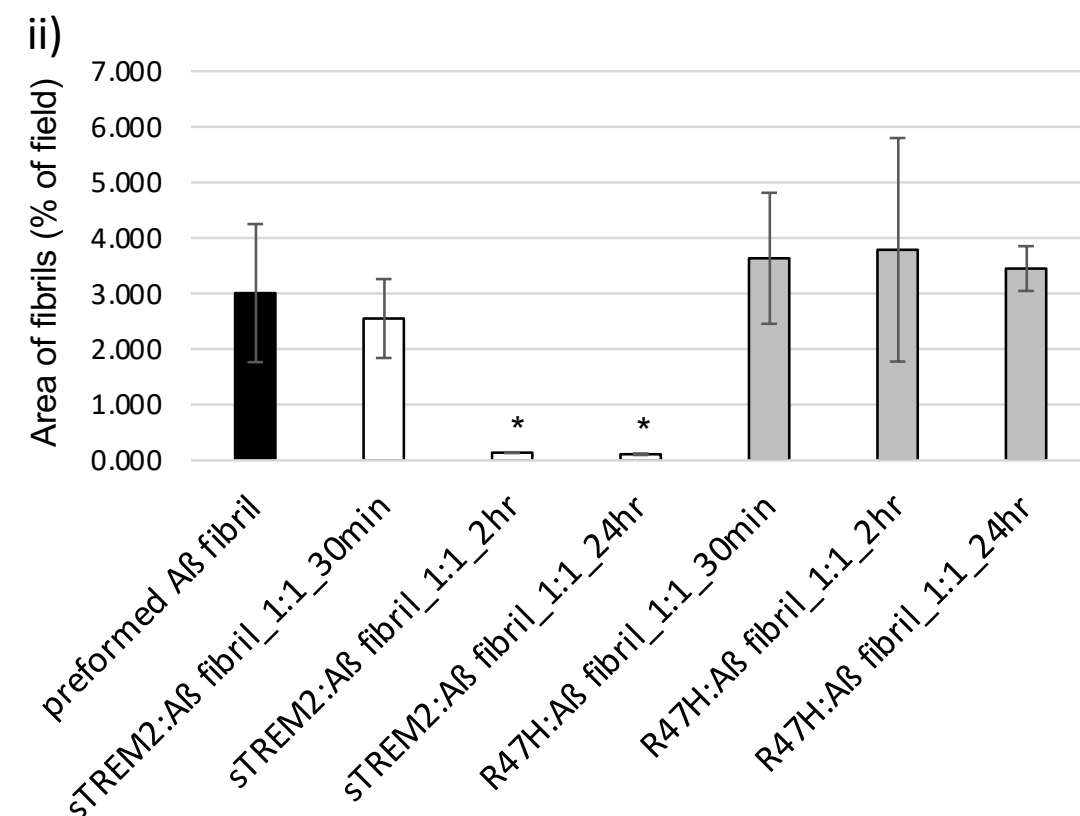

Supplementary Figure 12. WT sTREM2 disaggregates preformed A $\beta$ fibrils. Time course for experiment depicted in Fig. 3. i) Preformed $A \beta$ fibrils were treated \pm WT or R47H sTREM2 (at a 1:1 molar ratio) for $30 \mathrm{~min}, 2 \mathrm{hrs}$ or $24 \mathrm{hrs}$. Negative-stain TEM revealed that WT STREM2 dissociated $A \beta$ fibrils after 2 hour incubation but R47H did not. ii) Quantification of the area of $A \beta$ fibrils. Error bars represent SD. Statistical analysis was performed using one-way ANOVA followed by Bonferroni's multiple comparison test $\left(n=3-8,{ }^{*} p<0.05\right.$ vs pre-formed $A \beta$ fibril). 


\section{Supplementary Figure 13}
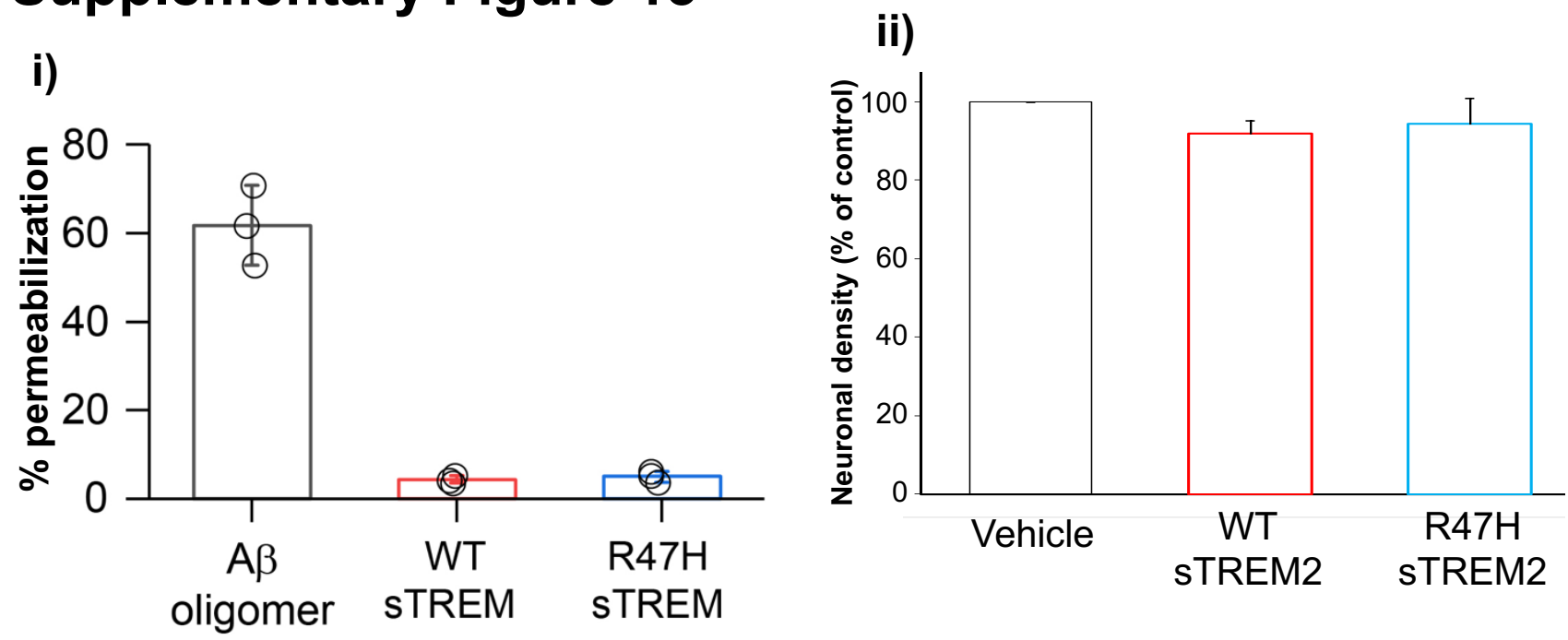

Supplementary Figure 13. In the absence of $A \beta$, wild-type and R47H sTREM2 have no effect on i) membrane permeability or ii) neuronal density. i) $1 \mu \mathrm{M} \mathrm{A \beta}$, WT sTREM 2 or R47H STREM2 were incubated (separately) for 6 hours and diluted to $200 \mathrm{nM}$ before the membrane-permeabilization assay was performed. Error bars $=S E M ; n=3$ independent experiments. ii) Mixed neuronal-glial co-cultures were treated with either: vehicle, $40 \mathrm{nM}$ wild-type STREM2 or $40 \mathrm{nM}$ R47H STREM2, and 3 days later neuronal density was counted. Error bars = SEM; $n=4$ independent experiments on different cell cultures. 